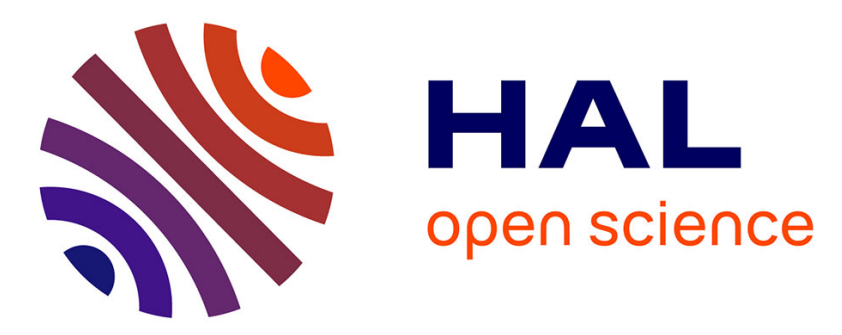

\title{
Dynamique du peuplement et activités agro-pastorales durant l'âge du Bronze dans les massifs du Haut Champsaur et de l'Argentierois (Hautes-Alpes)
}

Kevin Walsh, Florence Mocci, Mona Court-Picon, Stefan Tzortzis, Josep Maria Palet Martinez, Vincent Dumas, Vanessa Py, Maxence Segard, B. Talon

\section{To cite this version:}

Kevin Walsh, Florence Mocci, Mona Court-Picon, Stefan Tzortzis, Josep Maria Palet Martinez, et al.. Dynamique du peuplement et activités agro-pastorales durant l'âge du Bronze dans les massifs du Haut Champsaur et de l'Argentierois (Hautes-Alpes). Documents d'archéologie méridionale, 2005, 28, pp.25-44. hal-02128653

\section{HAL Id: hal-02128653 https://hal.science/hal-02128653}

Submitted on 14 May 2019

HAL is a multi-disciplinary open access archive for the deposit and dissemination of scientific research documents, whether they are published or not. The documents may come from teaching and research institutions in France or abroad, or from public or private research centers.
L'archive ouverte pluridisciplinaire HAL, est destinée au dépôt et à la diffusion de documents scientifiques de niveau recherche, publiés ou non, émanant des établissements d'enseignement et de recherche français ou étrangers, des laboratoires publics ou privés. 


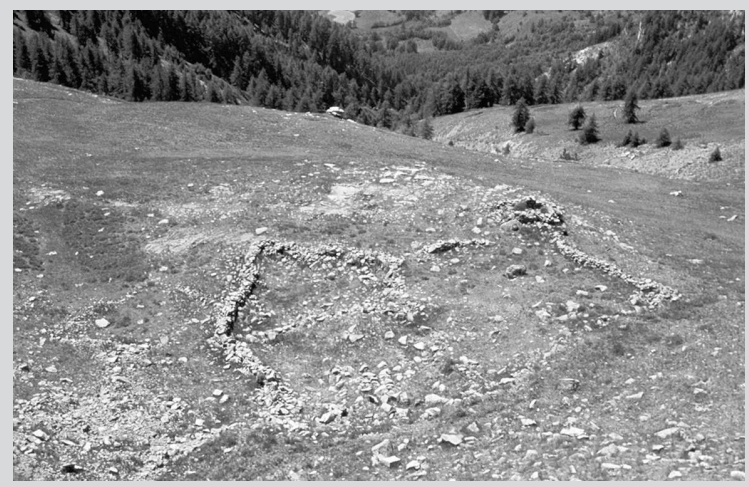

\title{
Dynamique du peuplement
}

et activités agro-pastorales

durant l'âge du Bronze

dans les massifs du Haut Champsaur et de 1'Argentierois (Hautes-Alpes)

\author{
Kevin WALSH*, Florence MOCCI**, Mona COURT-PICON*** \\ Stefan TZORTZIS $* * * *$ et Josep-Maria PALET-MARTINEZ $* * * * *$ \\ avec la collaboration de Vincent DUMAS, Vanessa PY, \\ Maxence SEGARD et Brigitte TALON
}

Des programmes de recherche pluridisciplinaires sur l'occupation du sol et le pastoralisme de la Préhistoire au Moyen Âge dans le sud du massif alpin sont menés, depuis 1998, sur les massifs du Haut Champsaur, de Freissinières et de l'Argentièrois (Hautes-Alpes). Des dix phases d'occupation et d'activité agropastorale mises en évidence (prospections pédestres et fouilles), entre 1600 et $2700 \mathrm{~m}$ d'altitude, trois se distinguent: la fin du Néolithique, l'âge du Bronze et la période médiévale. Au travers des premières données archéologiques et environnementales, cet article présente, depuis le milieu du $\mathrm{III}^{\mathrm{e}}$ millénaire au début du $\mathrm{I}^{\mathrm{er}}$ millénaire, les grandes caractéristiques de l'occupation du sol mais aussi l'originalité et l'importance de l'activité humaine dans cette zone alpine. La fin du Néolithique et l'âge du Bronze correspondent à une multiplication des gisements archéologiques marquant de façon évidente une rupture dans la gestion de l'espace montagnard. Les paysages sont largement façonnés par les activités humaines et l'entretien des terres cultivées, des prairies et des alpages, paraît continu. À la lumière des données de terrain, l'une des évolutions qui apparaît sur les sites d'altitude durant cette période concerne l'apparition de structures pastorales bâties entre 2067 et $2303 \mathrm{~m}$ d'altitude (datation ${ }^{14} \mathrm{C}$ ).

Mots-clés: occupation du sol, montagne, Néolithique, âge du Bronze, structure pastorale, anthracologie, palynologie, carbone 14 .
Since 1998, two multi-disciplinary research programmes have been running in the southern French Alps. Concentrating on the Haut Champsaur, Freissinières and the Argentières area, they study the settlement and economy from the prehistoric periods through to the middle ages. Ten phases of activity between 1600 and $2700 \mathrm{~m}$ have been identified as a result of prospection and excavation. However, three major phases of activity (often agro-pastoral in nature) stand out: the end of the Neolithic, the Bronze Age and the Medieval period. Integrating both archaeological and environmental evidence, this article presents the development of an original and intensely exploited landscape between the middle of the third millennium through to the beginning of the first millennium BC. The end of the Neolithic and the Bronze Age are characterised by an important increase in the number of sites that represent a change in the exploitation of the mountain zone. The landscape is affected by human activities that comprise the use of cultivated zones and the development of alpine pastures. One important development during this period is the appearance of pastoral structures between 2067 and $2303 \mathrm{~m}$ all dated by $14 \mathrm{C}$.

Keywords: settlement, mountains, Neolithic, Bronze Age, pastoral structures, anthracology, palynology, carbon 14. 


\section{Des prospections aux fouilles sur les massifs du Haut Champsaur et de l'Argentièrois}

$\mathrm{P}$ lusieurs Programmes Collectifs de Recherche pluridisciplinaires ${ }^{1}$ ont été développés depuis 1998 sur les activités humaines en moyenne et haute montagne dans les Alpes méridionales françaises et plus particulièrement sur sept communes de l'Argentièrois, de la Vallouise et du Haut Champsaur: Freissinières, L'Argentière-la-Bessée, Puy-Saint-Vincent, Vallouise, Champoléon, Orcières et Saint-Jean-Saint-Nicolas (fig. 1). La particularité de ces communes réside dans le fait qu'une grande portion de leur territoire, constitué essentiellement de hauts massifs alpins (entre 900 et 3200 mètres d'altitude), est intégrée dans la zone méridionale et orientale du Parc national des Écrins (département des Hautes-Alpes). Un milieu naturel assez varié caractérise ces espaces cloisonnés qui s'inscrivent au sein des unités orographiques du Drac, dans le Haut Champsaur, du torrent de la Byaisse, dans la vallée de Freissinières, des torrents du Fournel et de Narreyroux pour les zones d'alpage de l'Argentière-la-Bessée et de Puy-Saint-Vincent.

Les différentes opérations archéologiques programmées (prospections pédestres diachroniques, sondages et fouilles extensives) ont permis d'inventorier, entre 1600 et $2700 \mathrm{~m}$ d'altitude, plus de 260 sites datés entre le Mésolithique ancien ou moyen et la période moderne. Les sondages et fouilles ont concerné plus particulièrement, à ce jour, les

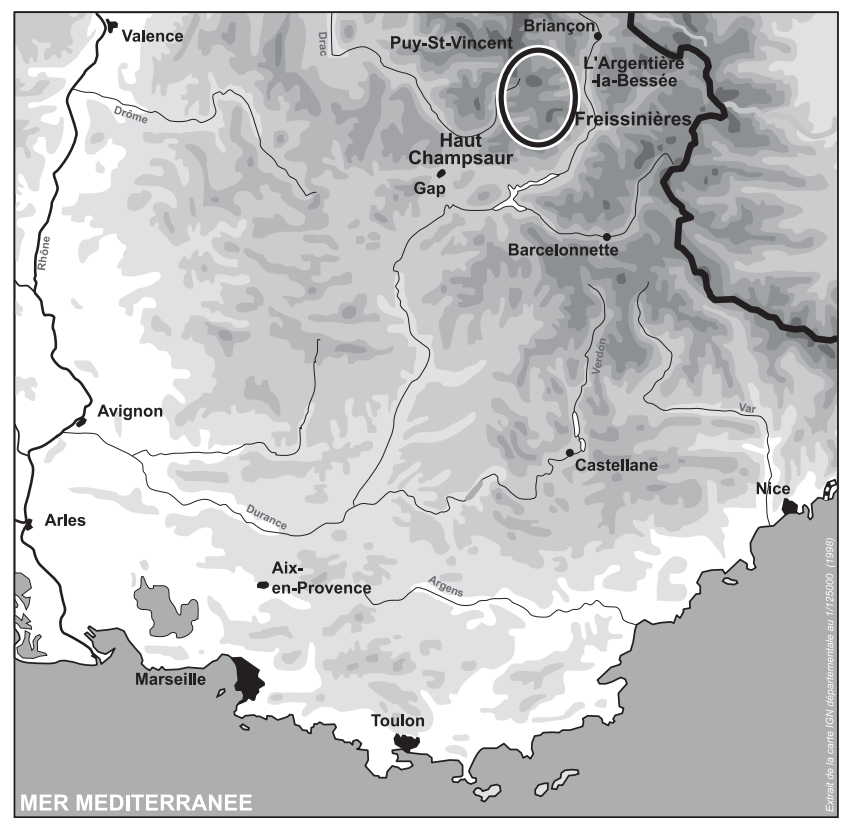

11 Situation des massifs de Freissinières et du Haut Champsaur (Hautes-Alpes, Parc national des Écrins). sites de moyenne et haute altitude de Freissinières et du Haut Champsaur sur lesquels se concentraient une série de gisements préhistoriques et de structures pastorales et agricoles de typologie et de période différentes ${ }^{2}$ (fig. 2). Le phénomène marquant de ces travaux réside dans l'absence de matériel archéologique d'époque historique mais aussi d'ossements (sols trop acides) alors que le matériel lithique est nettement présent, plus particulièrement sur les hauts plateaux de Freissinières, de l'Argentière-la-Bessée et de Puy Saint Vincent. Ainsi, la datation des vestiges étudiés s'est essentiellement appuyée sur l'analyse, par la méthode du carbone 14, des charbons de bois issus de foyers, de niveaux d'incendie ou d'écobuage recueillis à l'intérieur de cabanes ou d'enclos. Trente-huit sites sont ainsi précisément datés. Cette série recensée dans deux espaces différents correspond à un échantillonnage représentatif de l'occupation $\mathrm{du}$ sol en moyenne et haute montagne. Les phases de croissance dans l'activité humaine sont similaires et révèlent des traces de peuplement ou de fréquentation depuis le Paléolithique supérieur jusqu'à l'époque moderne ainsi qu'une pérennité dans le choix d'implantation de certains sites (buttes, replats, proximité de points d'eau), d'une période à une autre.

Le caractère même des traces d'occupation et du peuplement en milieu montagnard et la difficulté de déterminer une période chronologique spécifique a exigé une approche diachronique et systémique de cet espace (Leveau 2003; Palet-Martinez et al. 2003; Segard et al. 2003; Walsh, Mocci 2003a; Walsh 2005). Dans cette perspective, l'approche paléo environnementale a été privilégiée et a consisté en des prélèvements palynologiques sur certaines tourbières d'altitude ${ }^{3}$, des analyses anthracologiques des charbons de bois issus des fouilles ${ }^{4}$ et des analyses physico-chimiques des sédiments ${ }^{5}$. L'objectif des analyses palynologiques entreprises dans le cadre de ce travail était avant tout d'offrir, par l'étude d'enregistrements sédimentaires continus, une trame chronologique et un lien fédérateur aux autres disciplines, mais aussi d'appréhender sur la longue durée les dynamiques du milieu végétal et des manifestations anthropiques (Leveau, Beaulieu 2001; Beaulieu et al. 2003).

Des dix phases d'occupation mises en évidence sur ces hauts massifs, trois grandes phases d'activité agropastorale se distinguent: la fin du Néolithique, l'âge du Bronze et la période médiévale (à partir du VII s. ap. J.-C.). Au travers des premières données archéologiques et environnementales, cet article présente, depuis le milieu du III e millénaire av. n. è. au début du Irr millénaire ap. J.-C., les grandes caractéristiques de l'occupation du sol mais aussi l'originalité et l'importance de l'activité humaine dans cette zone sub-alpine et alpine où, jusqu'en 1998, «aucune structure d'habitat n'avait encore été découverte pour l'âge du Bronze » (Haussmann 1998, 97-135; Bocquet 2004a, 44-47). 


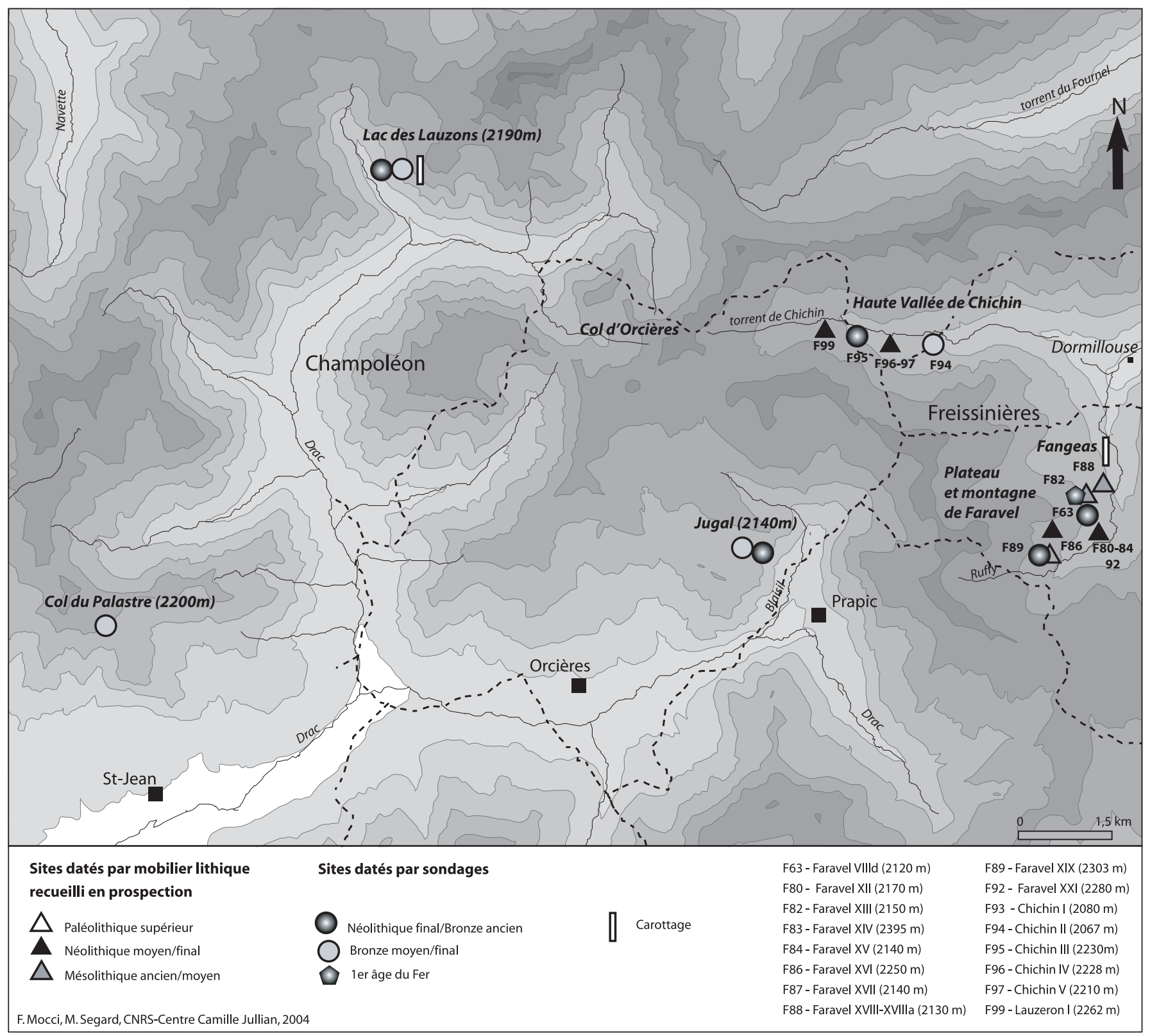

12 Carte des sites préhistoriques et protohistoriques découverts dans les massifs de Freissinières et du Haut Champsaur (2074-2 303 m).

\section{Les premières traces préhistoriques}

T es recherches de terrain menées entre 2100 et $2600 \mathrm{~m}$ Ld'altitude ont révélé, essentiellement sur les zones d'alpage de la commune de Freissinières (plateau et Montagne de Faravel, haute vallée de Chichin), de l'Argentière-laBessée (vallée du Haut Fournel) et de Puy-Saint-Vincent (massif des Grands Fonds), des traces de fréquentation non seulement au cours du Néolithique moyen et final mais aussi durant le Mésolithique ancien ou moyen de phylum épigravettien et le Mésolithique récent de type castelnovien (Mocci et $a l$. à paraître). Certaines données, extrêmement ténues mais néanmoins présentes, laissent également envisager un passage de groupes humains au cours du Paléolithique supérieur (site de Faravel XIX à 2303 m). La mise en évidence de vingt sites ou indices de site, en dépit d'une modestie certaine des corpus de vestiges recueillis, permet de compléter les connaissances relatives au peuplement préhistorique de la montagne issues notamment des travaux menés en France dans les Alpes françaises ${ }^{6}$ (Pion 1998; Bintz 1994 ; Bintz 2004, 38-39; Bintz, Bracco 2004, 34-35; Bintz, Morin 2001, 46; Bocquet 2004b, 40-41; Brochier et al. 1999; Morin 2000 et à paraître; Morin et al. 2004, 2005 ; Muret 1991) et dans la partie italienne ou valaisanne de l'arc alpin (Curdy 2002; Fedele 1990; Broglio 1994). 
Les traces de fréquentation au cours du Mésolithique, uniquement représentées pour l'instant sur le plateau et la Montagne de Faravel, avec les sites de plein air de Faravel XIII, XVIII et XVIIIa à 2130-2150 m d'altitude (fig. 2), constituent des découvertes importantes qui mettent en perspective une problématique relativement neuve, celle d'une datation relativement précoce des premières pénétrations en haute montagne dans les Alpes du Sud consécutivement à la fin des temps glaciaires (Bressy 2002, 66-86; Tzortzis 2001, 41-64 et 2002, 53-65; Walsh, Mocci 2003a; Brochier 2005,31 ). Les données dont nous disposons sur les fluctuations, l'extension maximale et le reflux de l'englacement würmien en Haute-Durance (Rousset et al. 1976; Jorda 1988; Jorda 1991) apparaissent finalement assez compatibles avec l'idée d'une arrivée jusqu'en haute montagne de groupes humains depuis les régions méridionales (Provence, Ligurie) par le sillon durancien dès la fin du tardiglaciaire et le début de l'Holocène. Cette fréquentation suggère très vraisemblablement la présence temporaire de groupes de chasseurs explorant, de façon saisonnière, le domaine de la haute montagne afin d'en éprouver les potentialités cynégétiques. Les sites et indices de site du Néolithique apparaissent toutefois à ce jour les plus nombreux (Faravel XII, XV, XVI, XVII, XXIII, XXII, Chichin II, IV, V, IX, X et Lauzeron I entre 2074-2264 m; dans la haute vallée du Fournel, Serre de l'Homme II, III, Petit Clausis II et Folie IV entre 1890-2240 m; dans le massif du Grand Fond, Grand Founze VII à 2614 m).

\section{De la fin du Néolithique à l'âge du Bronze : premiers témoignages d'exploitation et d'occupation}

L

a fin du Néolithique et l'âge du Bronze correspondent à une multiplication des gisements archéologiques, marquant de façon évidente une rupture dans la gestion de l'espace montagnard, sans doute à mettre en rapport avec l'essor démographique observé par l'archéologie, notamment dans les zones basses (Garcia 1995 ; Morin 2000). Les paysages sont largement façonnés par les activités humaines et l'entretien des terres cultivées, des prairies et des alpages paraît continu. À la lumière des données de terrain, l'une des évolutions qui apparaît sur les sites d'altitude entre la fin du Néolithique et le début de l'âge du Bronze concerne l'apparition de structures pastorales bâties. Sur l'ensemble de notre zone d'étude, sept structures implantées entre 2067 et 2303 m connaissent une occupation au cours de l'âge du Bronze ancien et moyen (datation ${ }^{14} \mathrm{C}$ ): dans le Haut Champsaur, Col du Palastre II à Saint-Jean-SaintNicolas (2200 m), Lac des Lauzons II à Champoléon $(2190 \mathrm{~m})$ et Jujal à Orcières $(2140 \mathrm{~m})$; dans la vallée de Freissinières, sur le plateau et la montagne de Faravel,
Faravel XIX (2303 m) et Faravel VIIId (2120 m) et dans la haute vallée de Chichin, Chichin II (2067 m) et Chichin III (2230 m).

Certains éléments architecturaux et topographiques caractérisent les gisements de cette période: construits en blocs de schiste non équarris, ils correspondent à des enclos, de forme triangulaire ou ovoïde de 20 à $150 \mathrm{~m}^{2}$ environ, sans aménagements internes et à des cabanes de forme ovoïde ou rectangulaire de superficie modeste (entre 3 à $10 \mathrm{~m}^{2}$ ) contenant des vestiges de foyers ou des niveaux d'incendie. Ces structures sont localisées sur des replats, en bordure de falaise ou dans des dépressions, à proximité de torrents, de lacs ou de tourbières. La topographie naturelle du terrain a été souvent exploitée afin d'asseoir les structures: soit en en prenant appui sur des blocs d'éboulis ou sur les comblements de ravines, soit en utilisant comme parements, d'énormes blocs erratiques déjà en place. Plus précisément, trois types d'aménagements ont été distingués et sont analysés dans les § 3.1. à 3.3.:

- un ensemble de structures contemporaines les unes aux autres correspondant à une surface enclose (Faravel XIXE2, Chichin II-E3, Chichin III-E1) attenant ou à proximité d'une ou plusieurs cabanes de berger (Faravel XIX-E1E3, Chichin II-E1, Chichin III-E2);

- une surface enclose insérée au sein de plusieurs structures pastorales de période et de forme différentes (Jujal I, Col du Palastre II);

- des structures totalement isolées tel l'enclos de Faravel VIIId ou la cabane du Lac des Lauzons II.

Dans l'état actuel des connaissances, le seul matériel archéologique recueilli dans les niveaux du Néolithique final/âge du Bronze ancien et moyen correspond à des objets lithiques (Faravel VIIId, Chichin II et Chichin III). Il s'agit de séries peu abondantes résultant pour l'essentiel d'une production de supports laminaires et lamellaires, de facture souvent soignée, avec quelques pièces façonnées (burin, perçoir/denticulé, troncature). Les principaux matériaux utilisés pour cette production, silex barémo-bédoulien et silex hauterivien, ont une provenance relativement lointaine: Haute Provence d'une part, sud-ouest du département des Hautes-Alpes d'autre part (Bressy 2002, 66-86).

Les datations ${ }^{14} \mathrm{C}$ réalisées à partir des foyers domestiques ou des niveaux d'incendie inhérents à des déboisements (en vue de la mise en valeur pastorale d'un secteur) révèlent une occupation de ces sept sites entre le milieu du $\mathrm{III}^{\mathrm{e}}$ millénaire et le début du Ir millénaire av. J.-C. ${ }^{7}$ (fig. 3). Dans la Haute vallée de Chichin, la présence de structures pastorales dès le milieu du $\mathrm{III}^{\mathrm{e}}$ millénaire (Chichin III) et sur le plateau de Faravel, la datation quasiment identique des deux sites du début du III ${ }^{\mathrm{e}}$ millénaire (Faravel XIX et Faravel VIIId) attestent une activité relativement forte de ces zones d'altitude à l'extrême fin du Néolithique. Cette fréquentation ancienne est confirmée dans le Haut Champsaur 
Atmospheric data from Stuiver et al. (1998); OxCal v3.9 Bronk Ramsey (2003); cub r:4 sd:12 prob usp[chron]

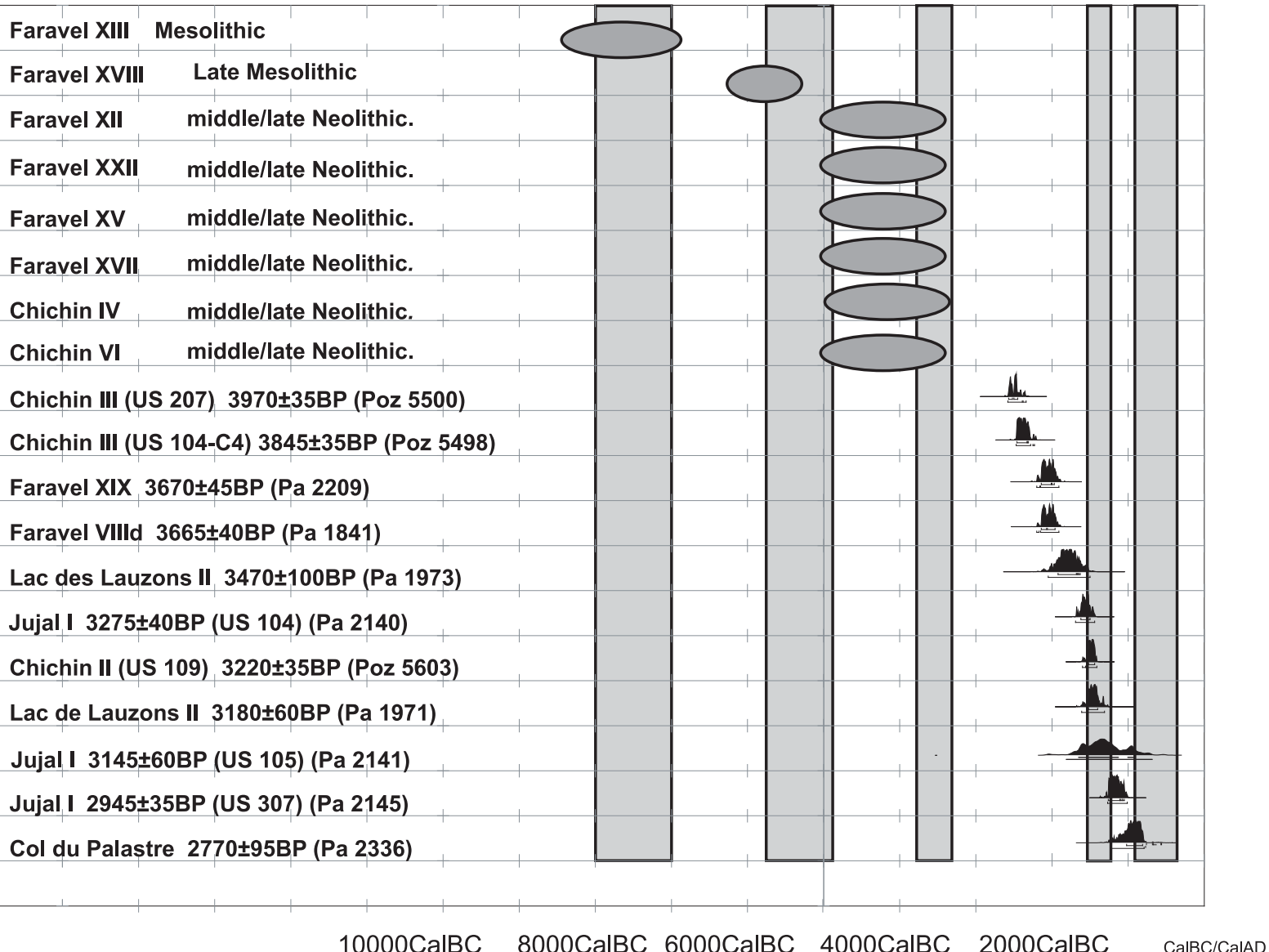

1. Datation carbone 14

$\bigcirc$ Présence de mobilier lithique
Péjoration climatique

Diagramme FI. Mocci, M. Segard, K. Walsh (Centre Camille Jullian - Université de York), 2005 avec la découverte, dans la cabane du Lac des Lauzons II, d'un sol d'occupation à la fin du II e millénaire avant notre ère. La datation d'un foyer plus récent indique une pérennité dans la fréquentation de la cabane au milieu du $\mathrm{II}^{\mathrm{e}}$ millénaire avant notre ère. À cette période de transition Bronze ancienBronze moyen peuvent être rattachées les structures pastorales de Chichin II et Jujal I. Sur ce dernier, les analyses anthracologiques ${ }^{8}$ ont révélé que plusieurs phases d'incendie avaient affecté le secteur avant et après la construction de l'enclos (cf. infra § 3.2.). Seul le site du col du Palastre II révèle une occupation au début du premier millénaire sur ces zones d'alpage.

Cette occupation continue de la haute montagne à la fin du Néolithique et durant l'âge du Bronze est illustrée par les données polliniques issues du Lac des Lauzons et analysées par M. Court-Picon ${ }^{9}$ (vallon d'Isola, Champsaur; Court-Picon
2003; Segard et al.2003). La haute résolution chronologique obtenue sur ce plateau met en évidence localement une continuité des activités agro-pastorales depuis le Néolithique jusqu'à nos jours (cf. infra $§ 4)$.

\subsection{LES ENSEMBLES PASTORAUX DE LA HAUTE VALLÉE DE CHICHIN ET DE LA MONTAGNE DE FARAVEL (MILIEU III ${ }^{\mathrm{e}}$ - MILIEU II ${ }^{\mathrm{e}}$ MILLÉNAIRE)}

Dans la Haute Vallée de Chichin (fig. 2-4), le site de Chichin III est à ce jour le plus ancien aménagement pastoral de la fin du Néolithique/âge du Bronze ancien identifié sur cette partie du Parc national des Écrins (Walsh, Mocci 2003b). Implanté à $2230 \mathrm{~m}$ d'altitude, dans une petite dépression surplombant le torrent de la Grande Eau, il est constitué 

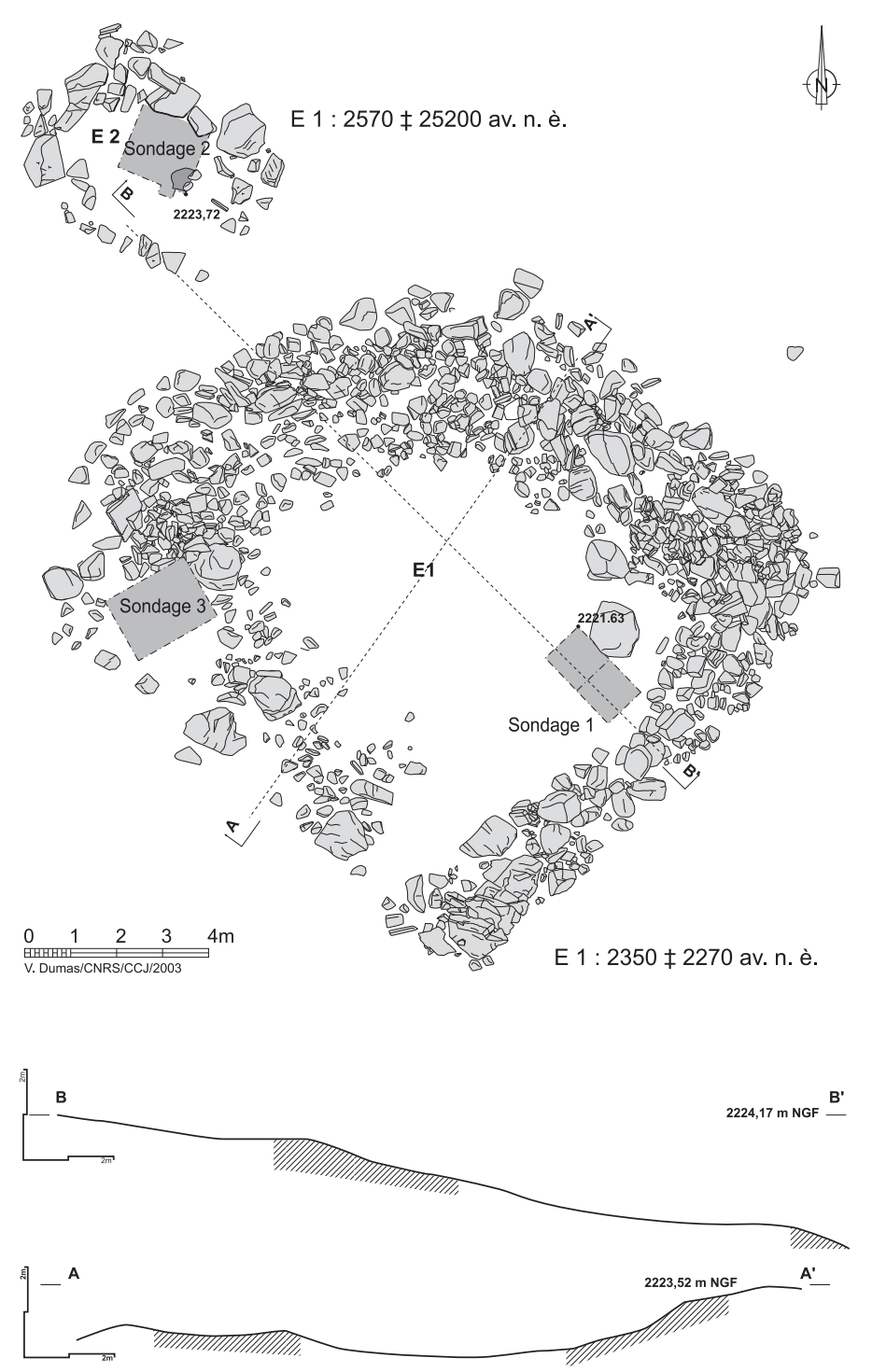

relevé des sections du site de Chichin II

I Relevé des sections et relevé pierre à pierre de l'ensemble des vestiges de la cabane et de l'enclos du site de Chichin III à 2230 m d'altitude (commune de Freissinières).

d'un vaste enclos (E1) entouré de petites cabanes dont une seule a été en partie fouillée (E2). Contrairement aux autres sites pastoraux reconnus sur le massif (Faravel XIX et Chichin II), l'enclos et l'unité domestique ne sont pas attenants mais sont éloignés de 4 à $5 \mathrm{~m}$ l'un de l'autre: au sud-est, dans une petite dépression nord-ouest/sud-est, un enclos de forme ovoïde d'une superficie totale de $162 \mathrm{~m}^{2}$ environ dont les murs en blocs de schiste non équarris sont visibles sur plusieurs assises (surface interne: $60 \mathrm{~m}^{2}$ ); au nordouest, sur un petit replat, une cabane rectangulaire de $22 \mathrm{~m}^{2}$ en grande partie enfouie (surface interne: $8,75 \mathrm{~m}^{2}$ ).

L'aménagement de l'enclos E1 s'est appuyé en partie sur des gros blocs erratiques déjà en place. La largeur des parois de cet enclos varie de 1 à $3 \mathrm{~m}$. L'accès à cet espace était situé au sud-ouest (largeur supposée : environ $3 \mathrm{~m}$ ). Six unités stratigraphiques ont été identifiées sur une épaisseur de $70 \mathrm{~cm}$. Elles révèlent essentiellement des phases de colluvionnement antérieures ou postérieures à l'utilisation de l'enclos mais surtout un paléosol caractérisé par une matrice argilo limoneuse compacte de couleur gris clair (matière humique) ${ }^{10}$ dans lequel a été recueilli un petit éclat cortical.

Dans la cabane E2, le parement interne nord-est mis au jour sur une à deux assises irrégulières (20 à $60 \mathrm{~cm}$ de haut), repose sur le substrat morainique. La largeur conservée des parements varie de 40 à $80 \mathrm{~cm}$. L'accès à cette cabane était situé au sud-est (largeur supposée: $2 \mathrm{~m}$ ). Neuf unités stratigraphiques ont été identifiées sur une épaisseur de $55 \mathrm{~cm}$. $\mathrm{Ce}$ site a des caractéristiques très similaires à la zone "domestique" du site de l'âge du Bronze de Faravel XIX (Walsh 2002). L'élément archéologique majeur réside dans la découverte partielle d'une surface limoneuse très rubéfiée de forme ovoïde de 1,60 $\mathrm{m}^{2}$ qui témoigne de la présence d'un foyer. Reposant sur le substrat morainique, cette surface a été mise au jour à $45 \mathrm{~cm}$ de profondeur, à l'extrémité sudest du sondage, dans la partie médiane de la cabane. Une couche charbonneuse relativement mince était repartie sur toute la limite est du sondage. Cette surface était en contact avec un sol d'occupation sur lequel reposaient quelques petites pierres posées à plat. Sur ce sol a été recueilli un seul éclat de silex ayant subi une forte altération thermique. La datation du foyer de la cabane a été estimée à 2460-2200 av. J.-C. (3845 \pm 35 BP; Poz-5498) et celle du paléosol de l'enclos, à 2580-2400 av. J.-C. (3970 \pm 35 ; Poz-5500).

L'étude anthracologique réalisée par Brigitte Talon révèle que les essences présentes sont le pin cembro, le mélèzeépicéa, le genévrier et le bouleau. La présence du bouleau et du genévrier, tous deux héliophiles, indique un milieu forestier peu dense. Le genévrier est un arbuste à port plus ou moins couché (Juniperus nana) que l'on trouve de préférence sur les versants bien exposés. Son bois, très résineux, en fait un allume feu de choix (comparé aux éricacées par exemple). Le bouleau est une essence très exigeante en lumière et dans le cas du bouleau pubescent, très exigeante en eau. Ce sont des arbres que l'on trouve donc fréquemment en bordure des cours d'eau ou des tourbières. Aucune de ces essences n'étant présente actuellement sur le site, soit le déboisement de cette zone par l'homme est contemporain ou postérieur à la fréquentation du site, soit les arbres montaient jusqu'à l'altitude de $2230 \mathrm{~m}$ au moins sur les versants sud (Talon 2003, 64-69).

Sur la Montagne de Faravel (fig. 2-5), le site de Faravel XIX est une petite cabane ovoïde dont les murs correspondent à des amas organisés, sans liant, de blocs de schiste non équarris, enserrant une superficie interne de $9 \mathrm{~m}^{2}$ environ (E1). Localisée sur un replat à une centaine de mètres en aval des trois terrasses du site de Faravel XX, cette cabane 


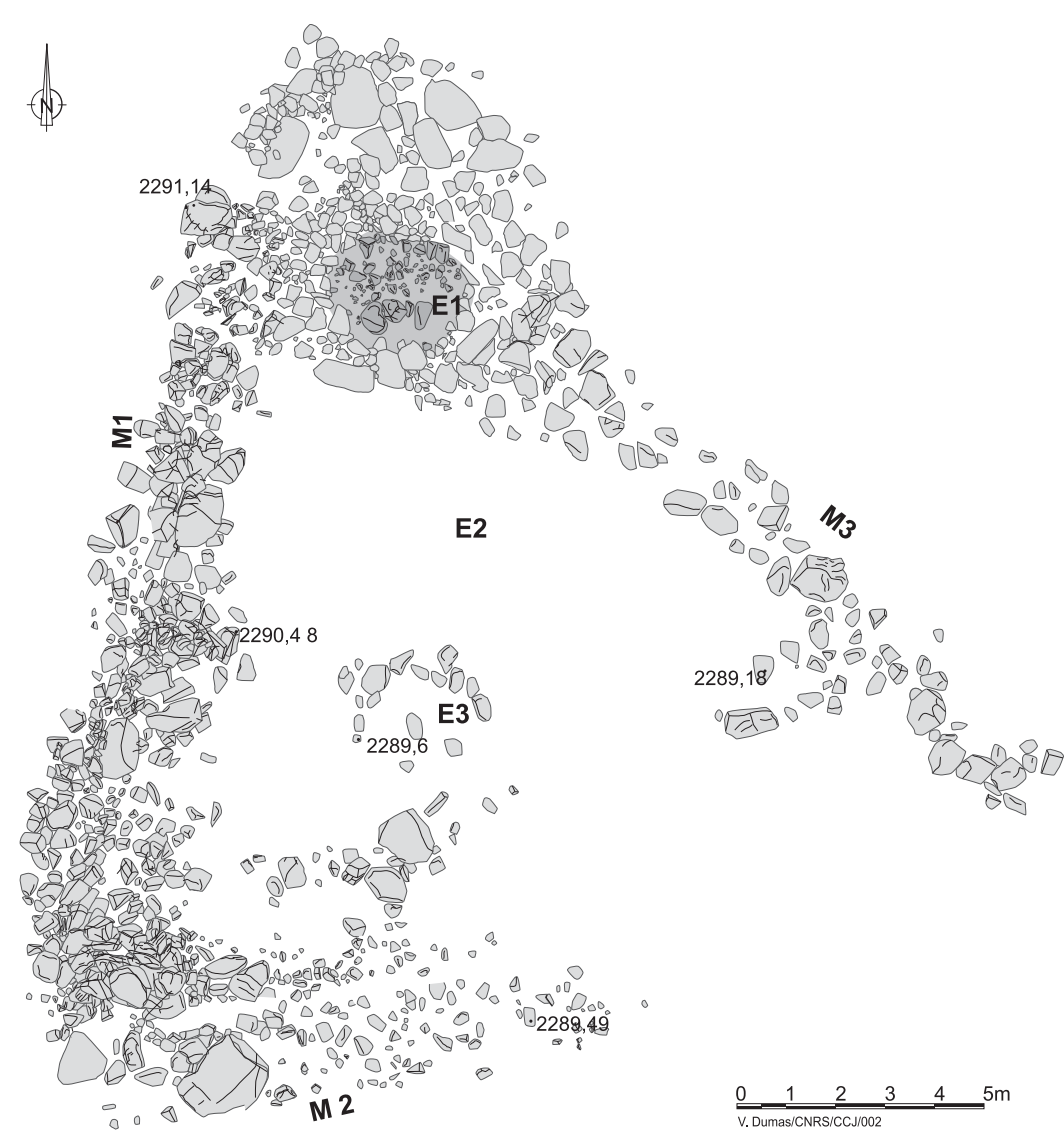

15 Relevé pierre à pierre de l'ensemble des vestiges du site de Faravel XIX à 2303 m d'altitude (Montagne de Faravel, Freissinières, 05).

n'est pas isolée: des amas de blocs structurés délimitent à l'ouest (M1), au sud (M2) et au nord-est (M3), depuis les parements sud de la cabane, une petite terrasse triangulaire d'une superficie de $100 \mathrm{~m}^{2}$ environ, qui a sans doute servi en tant qu'enclos (E2). À l'extrémité sud de cet enclos, la partie nord d'une petite structure circulaire est partiellement visible au sol (E3). Le décapage a concerné la quasi totalité de l'intérieur de cette structure soit une superficie de $6 \mathrm{~m}^{2}$ environ, dans les zones médiane et occidentale de la structure (Walsh 2002). Huit unités stratigraphiques ont été identifiées au cours de la fouille sur une épaisseur maximale de $30 \mathrm{~cm}$ mais aucune trace d'aménagement n'a été mise au jour. En revanche, deux états ont été identifiés: l'état I révèle des traces d'occupation comportant de rares nodules de charbons (US 103) mais aucun matériel archéologique n'a été recueilli. L'état II correspond à niveau d'occupation avec des vestiges d'un foyer résiduel (US 107) partiellement identifié sous des niveaux de destruction et établi sur le substrat morainique. Ce foyer, localisé au fond de la cabane, contre la paroi ouest, a été daté entre 2150 et 1920 av. J.-C. (3670 \pm 45 BP; Pa 2209).
L'analyse anthracologique réalisée par Vanessa Py (fig. 6) signale l'omniprésence du Pin cembro (Pinus cembra) malgré le caractère peu représentatif des prélèvements (dépôt ponctuel). À ce propos, il n'est pas inutile de rappeler sa rareté actuelle sur le secteur où les quelques stations connues sont à l'état de relique. Sa désertion précoce des vallées intraalpines a bien été démontrée par les études pédoanthracologiques récentes (Talon 1997; Talon et al. 1998). Elle a été amorcée et accrue jusqu'à son paroxysme par l'action anthropique (artisanat, industries, agro-pastoralisme; Py 2002). Les résultats anthracologiques offrent l'image d'un territoire d'approvisionnement en bois de feu composé de forêts (hautes futaies) et de pré-bois (formation végétale constituée d'une mosaïque d'éléments forestiers, prairiaux, d'ourlets et de manteaux) dominé exclusivement par la cembraie. Le caractère ponctuel du dépôt peut expliquer l'absence, dans le cortège floristique, des petits ligneux secondaires comme le Rhododendron ferrugineux (Rhodendron ferrugineum) ou les airelles. Les quelques indices de Pin à crochets (Pinus uncinata) pourraient refléter l'image de quelques bouquets d'arbres perchés audessus de ravins ventés ou croissant dans des zones tourbeuses et marécageuses, comme, par exemple, dans le vallon de Fangeas.

À $1500 \mathrm{~m}$ à l'est de Chichin III, à 2074 m d'altitude (fig. 2-7-8), le site de Chichin II est sans doute le gisement le plus original du secteur de Freissinières de par ses caractéristiques architecturales et la présence d'objets lithiques dans un niveau d'occupation daté de l'âge du Bronze moyen (Walsh, Mocci 2003b). Le site possède trois éléments distincts: une cabane ronde E1 $\left(4,40 \mathrm{~m}^{2}\right)$, une cabane carrée E2 $\left(2,10 \mathrm{~m}^{2}\right)$ et une surface enclose constituée d'alignements de blocs structurés délimitant une petite terrasse triangulaire d'une superficie de $230 \mathrm{~m}^{2}$ environ (E3). L'analyse stratigraphique et les vestiges archéologiques témoignent au moins

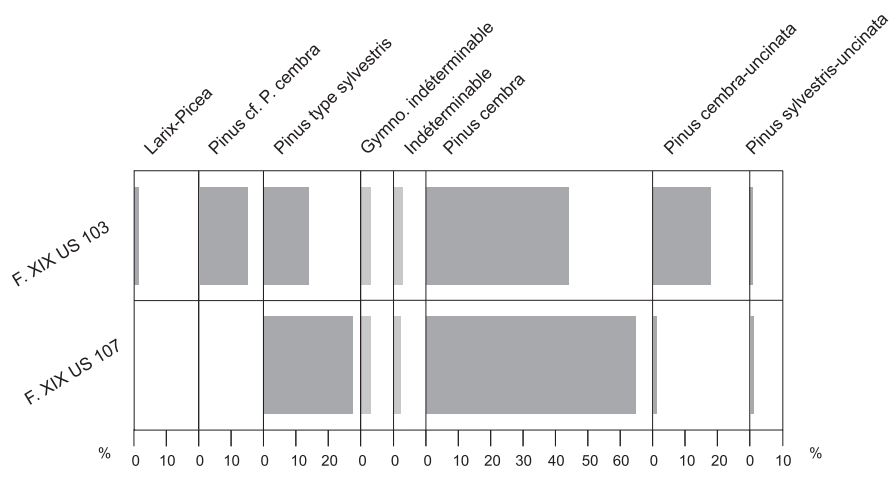

16 Diagramme anthracologique exprimé en fréquences relatives (\%) des US 103 et 107 du site de Faravel XIX (V. Py, 2003). 


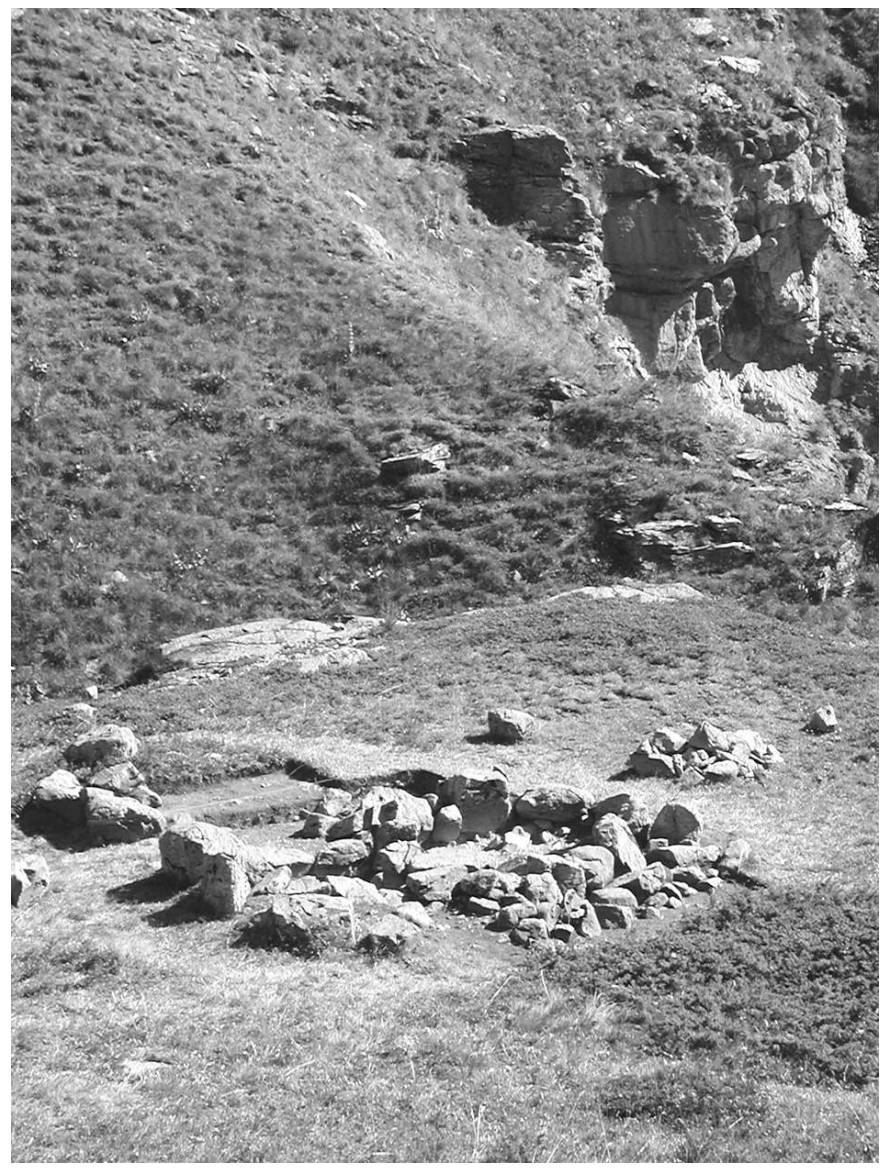

17 Vue depuis le sud-est des cabanes et de l'enclos de Chichin II à 2074 m d'altitude (haute vallée de Chichin, Freissinières, 05). Cliché K. Walsh, juillet 2003.

de deux phases d'occupation et d'aménagement (âge du Bronze moyen pour la cabane ronde, $\mathrm{XIV}^{\mathrm{e}} \mathrm{s}$. de notre ère pour la cabane carrée). Ces aménagements sont à quelques mètres plus au sud d'une vingtaine de cabanes rectangulaires médiévales/modernes construites dans des buttes morainiques.

La cabane circulaire E1 est constituée de quatre murs correspondant à des amas de blocs de schiste non équarris et sans liant. Les parements internes ont été mis au jour sur une à deux assises sur 40 à $70 \mathrm{~cm}$ de haut jusqu'au substrat morainique sur lequel ils reposent. La construction de cette structure s'est appuyée en partie sur des gros blocs erratiques déjà en place (parement ouest) alors que les autres parements, plus organisés, comportent des blocs de dimension moins importante (entre $20 \mathrm{~cm}$ et $80 \mathrm{~cm}$ ) mais aussi des plaquettes de lauzes. La largeur des murs varie de 53 à $93 \mathrm{~cm}$ hormis celle du mur sud $(1,19 \mathrm{~cm})$. L'accès à cette cabane n'a pu être déterminé. Le décapage a concerné l'intérieur de cet espace. Onze unités stratigraphiques ont été identifiées au cours de la fouille sur une épaisseur maximale de $78 \mathrm{~cm}$. Deux phases d'occupation ont été mises en évidence. La plus ancienne (état I), contemporaine de l'aménagement de la cabane, a été identifiée à $50 \mathrm{~cm}$ de profondeur environ. Elle correspond à un sol constitué d'un sédiment argilo-limoneux relativement compact (Us 109) qui repose directement sur la surface du substrat morainique. Sur ce sol ont été recueillis 34 objets lithiques ainsi que des nodules charbons de bois qui témoignent d'un reliquat de foyer ponctuel daté entre 1540-1410 av. J.-C. (3220 $\pm 35 \mathrm{BP}$; Poz-5603). Après une période d'abandon, plusieurs phases de colluvionnement se succèdent colmatant une destruction partielle des lauzes incluses dans les parois des murs. Cet espace est réoccupé (niveau de circulation en lauzes) sans doute lors la construction de l'Espace 2. L'enclos de Chichin II (E3), contigu avec le mur sud-ouest de l'Espace 2, a probablement été intégré lors de la construction de la cabane médiévale comme le mur nord-est de la cabane de l'âge du Bronze.

Le mobilier lithique, recueilli en grande partie dans la partie interne de la cabane circulaire (US 109, E1) et au nord de celle-ci, à l'extérieur (E3), a été analysé par Stéfan Tzortzis (fig. 8). L'unique nucléus de la série, en silex hauterivien, est un exemplaire de petite taille (env. $3 \times 3 \mathrm{~cm}$ ), à plan de frappe unique. Cette pièce est clairement orientée vers la production de lamelles dont cinq négatifs sont observables sur la table de débitage. Cette donnée corrobore la présence dans l'assemblage de lamelles ou de fragments lamellaires majoritairement en silex hauterivien et de petite taille. La présence de produits lamellaires et laminaires de belle facture, en silex barémo-bédoulien, dont certains ont été retouchés selon diverses modalités, doit être soulignée. Deux lames en particulier, bien standardisées, en silex bédoulien blond légèrement patiné, se situent visiblement dans la même phase de plein débitage et ont pu être remontées ensemble. Un nombre réduit d'outils sont présents mais ils sont néanmoins très intéressants du point de vue typologique: un burin multiple, un bec ou perçoir, une encoche retouchée sur éclat laminaire, un fragment mésio-proximal de lame à bord abattu et un grattoir mince réalisé sur un fragment mésio-distal de lame retouchée, ces deux dernières pièces constituant un seul et même support laminaire cassé dans sa partie mésiale. En dépit de l'absence d'armatures microlithiques, la technique du microburin est attestée par la présence d'un exemplaire distal en silex barémo-bédoulien. En conclusion, l'assemblage lithique évoque plutôt une ambiance néolithique, en particulier de part le mode de production laminaire soigné évoqué et si l'on admet que la technique du microburin, qui apparaît sporadiquement au Paléolithique supérieur et connaît sa pleine expansion au Mésolithique, se rencontre encore ponctuellement au Néolithique (Tzortzis 2003, 52-63). Dans l'état actuel des connaissances, nous ne pouvons néanmoins exclure le fait que ce matériel pourrait être contemporain de l'occupation de la cabane E1, le silex continuant d'être exploité bien après la fin du Néolithique (Ghesquière, Marcigny 1997, 27-47; Pons, Largarrigue 2003, 7-41). Pour autant, les caractéristiques du débitage, la nature des produits 

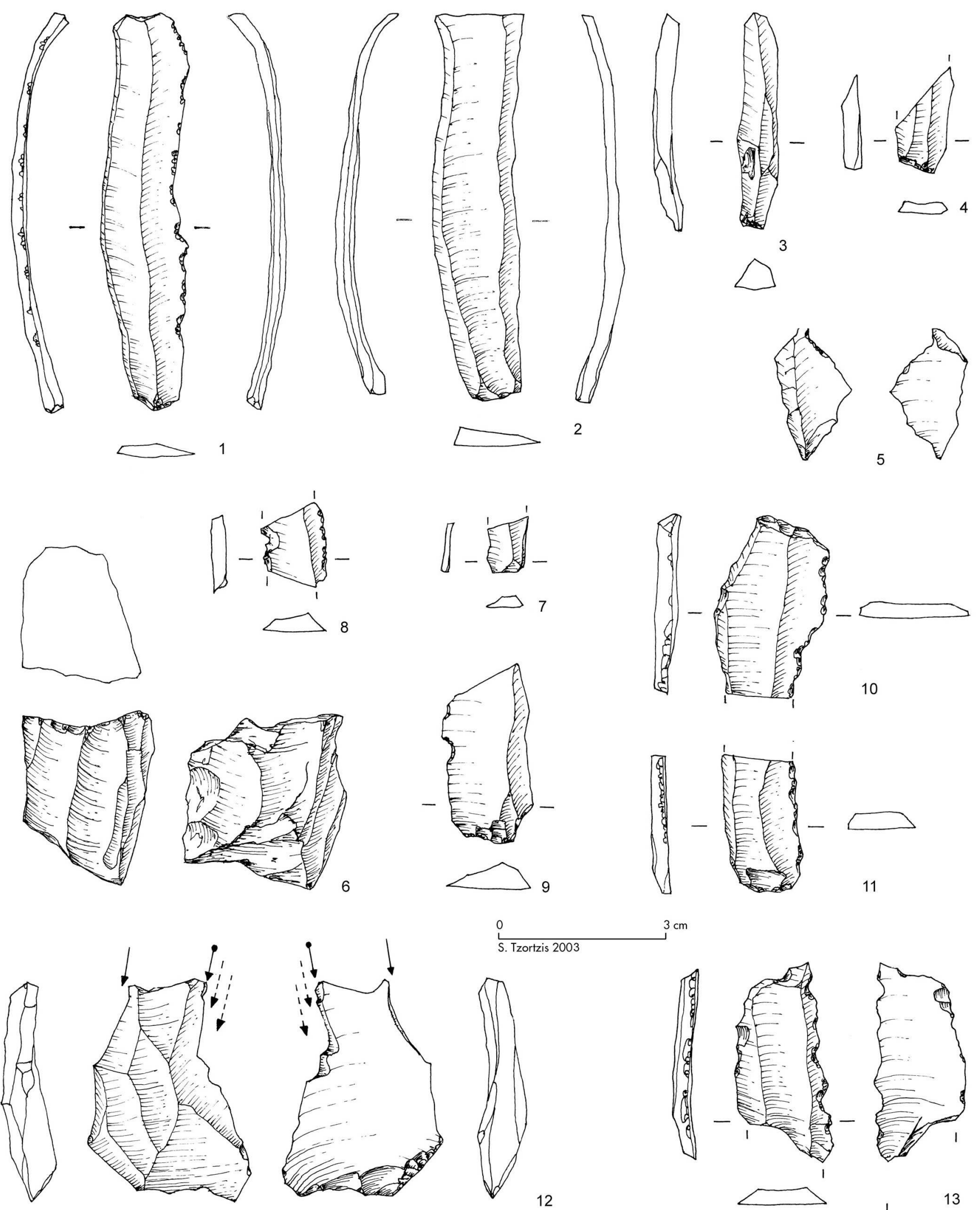

$3 \mathrm{~cm}$

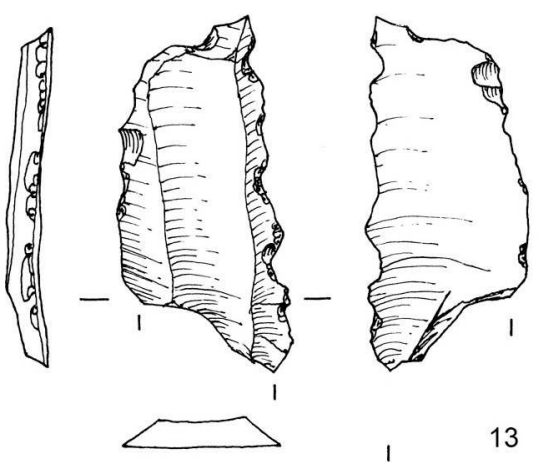

18 Mobilier lithique recueilli dans I'US 109 de l'Espace 1 du site de Chichin II (haute vallée de Chichin, commune de Freissinières) (S. Tzortzis). 

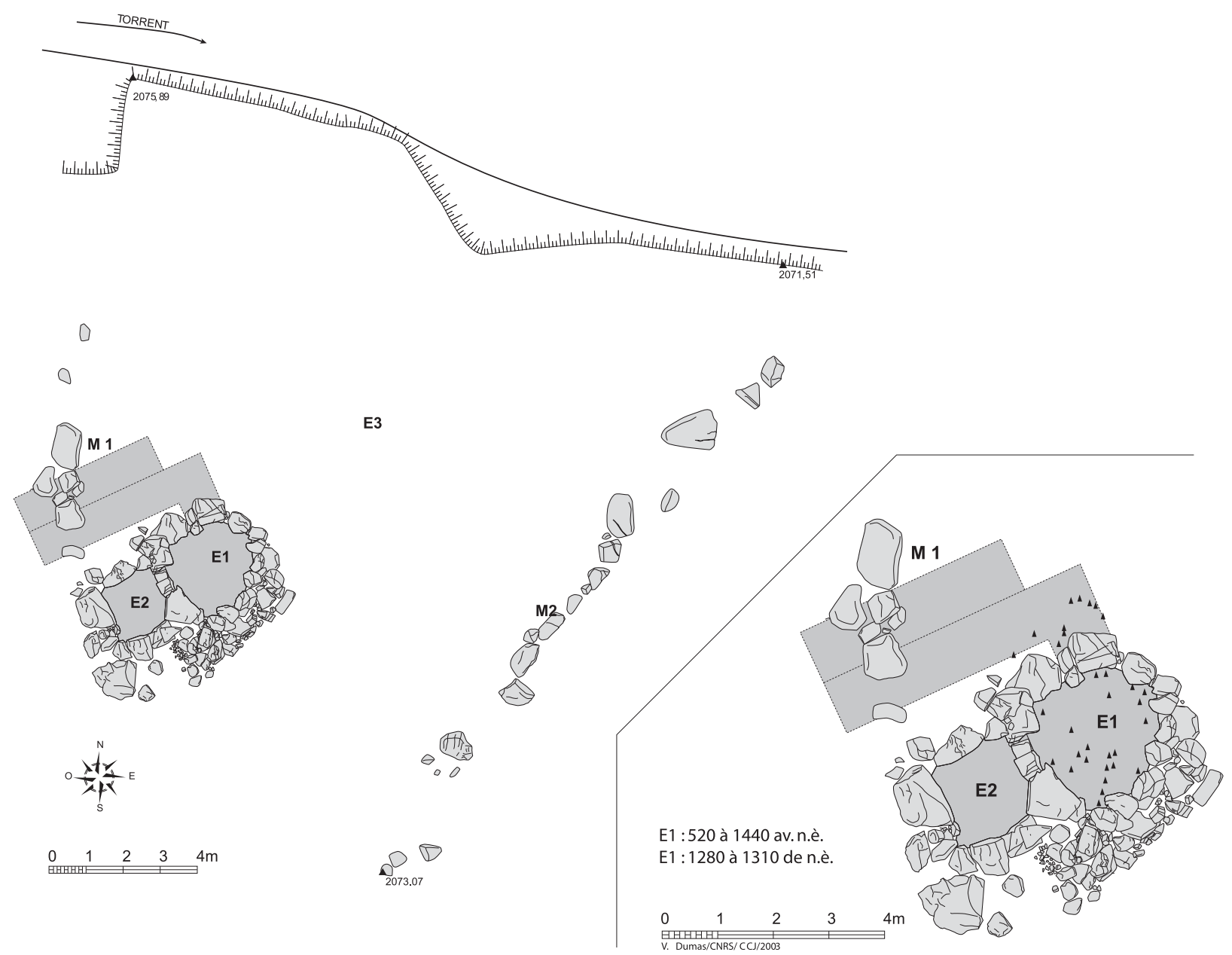

19 Relevé pierre à pierre de l'ensemble des vestiges du site de Chichin II et répartition du mobilier lithique dans l'Espace 1 (haute vallée de Chichin, commune de Freissinières).

lithiques, tout comme les modalités d'approvisionnement en matériau lithique semblent exclure l'idée d'un usage purement opportuniste et ponctuel de ce type de matière première. Il faudrait alors admettre, au contraire, que le silex constitue chez ces groupes humains, pour des raisons qui restent à déterminer, une ressource encore prépondérante, dans un environnement où les gîtes de cuivre ne font pourtant pas défaut et dont au moins l'un d'entre eux, les Clausis à Saint Véran dans le Queyras, est en exploitation dès le Chalcolthique ou le Bronze ancien (Barge et al. 1998).

L'analyse anthracologique du reliquat de foyer réalisée par Brigitte Talon révèle cinq à huit taxons parmi les 118 charbons observés: Pinus cembra, Larix/Picea, Juniperus sp., Betula, Acer (cf. opalus), Rhododendron ferrugineum, Vaccinium myrtillus, Salix $s p$. Les petits ligneux prennent de l'importance, en réponse probablement à l'ouverture du milieu. Ainsi, le genévrier, le rhododendron, les myrtilles sont-ils mieux représentés. Il est intéressant de constater aussi que le mélèze l'emporte sur le pin cembro de façon significative dans le paléosol de l'enclos. Le bouleau, l'érable et le saule témoignent de la proximité du torrent, le mélèze, le genévrier et le pin de type sylvestre d'un milieu ouvert de conditions plus xériques, le pin cembro, le rhododendron et les myrtilles d'un sol plus acide, avec un humus de type moder.

Cette mosaïque de végétation traduit assez bien la mosaïque des milieux exploités par l'homme pour la récolte du bois. Hormis le mélèze et les saules, aucune des essences identifiées n'est présente sur le site. L'érable et le pin de type sylvestre sont présents dans l'étage montagnard, dans les formations caducifoliées pour le premier et dans la pinède d'adret sur lande à genévrier pour le second. Les hypothèses suivantes peuvent être proposées: soit le déboisement de cette zone par l'homme est contemporain ou postérieur à la fréquentation du site, soit les arbres montaient jusqu'à l'altitude de $2067 \mathrm{~m}$ en versant sud (Talon 2003, 64-69). 


\subsection{LES ENCLOS DU HAUT CHAMPSAUR} (MILIEU II ${ }^{\mathrm{e}}$ - DÉBUT I ${ }^{\text {er }}$ MILLÉNAIRE)

L'enclos de Jujal, d'une superficie de $150 \mathrm{~m}^{2}$, est implanté au sud d'un vaste replat occupé par plusieurs structures pastorales de différentes périodes, dominant à $2200 \mathrm{~m}$ la haute vallée du Drac. Les sondages ont permis de situer le début de l'occupation de cet enclos au milieu du II e millénaire av. n. è. et une réoccupation sans doute à la période médiévale. L'analyse de la stratigraphie et des données anthracologiques révèle que le secteur a été affecté par plusieurs phases d'incendie, antérieures puis postérieures à la construction de l'enclos. Ces événements correspondent à un défrichement par le feu en vue de l'exploitation pastorale du secteur. La première phase d'incendie, identifiée dans deux sondages distants d'une dizaine de mètres environ (Us 105 et US 307), est constituée d'un niveau de cailloutis très riche en charbons de bois sur lequel a été directement construit l'enclos de Jujal. Les datations ${ }^{14} \mathrm{C}$ permettent de dater cet incendie pour l'Us 105, vers 1530-1250 av. J.-C. (3145 $\pm 60 \mathrm{BP}$; Pa 2141) et vers 1690-1440 av. J.-C., pour l'Us 307 (3275 \pm 40 BP; Pa 2140). Ces événements interviennent à la même période que la seconde occupation au Lac des Lauzons II (cf. infra § 3.3.). Le second incendie, daté entre 1270 et 1010 av. J.-C. (2945 \pm 35 BP; Pa 2145), correspond sans doute à une phase d'entretien de l'espace pastoral. La forêt, suffisamment régénérée depuis le premier incendie, empêchant le développement des herbacées indispensables au pâturage, l'emploi du feu s'est alors révélé nécessaire pour éclaircir la forêt ou ouvrir de nouvelles clairières.

L'enclos du Col du Palastre II est situé sur un replat du versant méridional du massif du Palastre (fig. 2-10). Sa forme et sa dimension sont à ce jour inconnues car il a été recouvert par un enclos polygonal de $600 \mathrm{~m}^{2}$ vraisemblablement

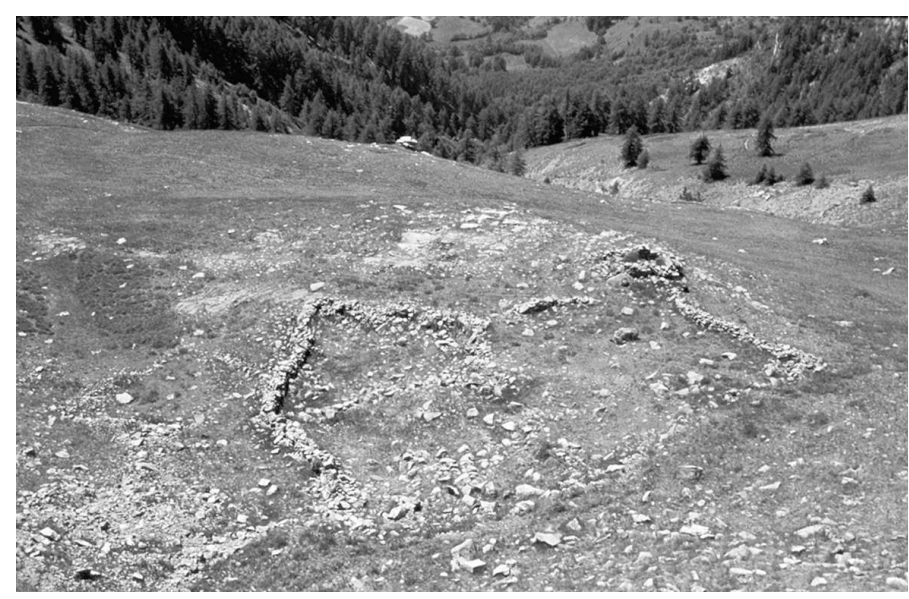

10 Vue depuis le nord des enclos et des cabanes du site du Palastre à $2200 \mathrm{~m}$ d'altitude (Saint-Jean-Saint-Nicolas). Cliché J. Palet-Martinez, juillet 1999. moderne associé à une cabane rectangulaire de l'époque moderne (XVIII ${ }^{\mathrm{e}}$-XIX ${ }^{\mathrm{e}} \mathrm{s}$.).

Les phases d'occupation les plus anciennes se situent entre 1220 et 790 av. J.-C. $(2770 \pm 95 \mathrm{BP} ; \mathrm{Pa} 2236)$ et puis à l'époque romaine (Palet-Martinez, Segard 2002).

\subsection{DES STRUCTURES PASTORALES ISOLÉES SUR LES PLATEAUX DU LAC DES LAUZONS ET DE FARAVEL (FIN III ${ }^{e}$ - MILIEU II ${ }^{e}$ MILLÉNAIRE)}

La cabane du Lac des Lauzons II est implantée sur un vaste replat dans la vallée d'Isola sur lequel a été dénombré un ensemble de huit cabanes et enclos établis autour d'une tourbière (fig. 2-11). Néanmoins, cette structure est relativement isolée des autres vestiges protégés par une moraine frontale qui s'étend au-dessus du gisement. La typologie, la distribution et l'état de conservation des aménagements sur ce plateau suggèrent que l'espace a été fréquenté jusqu'à l'époque moderne (Palet-Martinez 2000). La fouille de cette cabane, de forme ovoïde de $12 \mathrm{~m}^{2}$, a permis d'identifier deux phases successives d'occupation au cours de l'âge du Bronze avec une réoccupation sans doute à la période médiévale. La phase plus ancienne a été révélée par un sol d'occupation daté entre 2050 et 1500 av. J.-C. (3470 \pm 100 BP; Pa 1973) et par un foyer délimité par un cercle de petits blocs (1610-1310 av. J.-C; $3180 \pm 60$ BP; Pa 1971). Ce foyer correspond vraisemblablement à la dernière phase d'utilisation de cette cabane comme le confirme l'analyse anthracologique (variété d'espèces indicatives révélant une longue période d'activité).

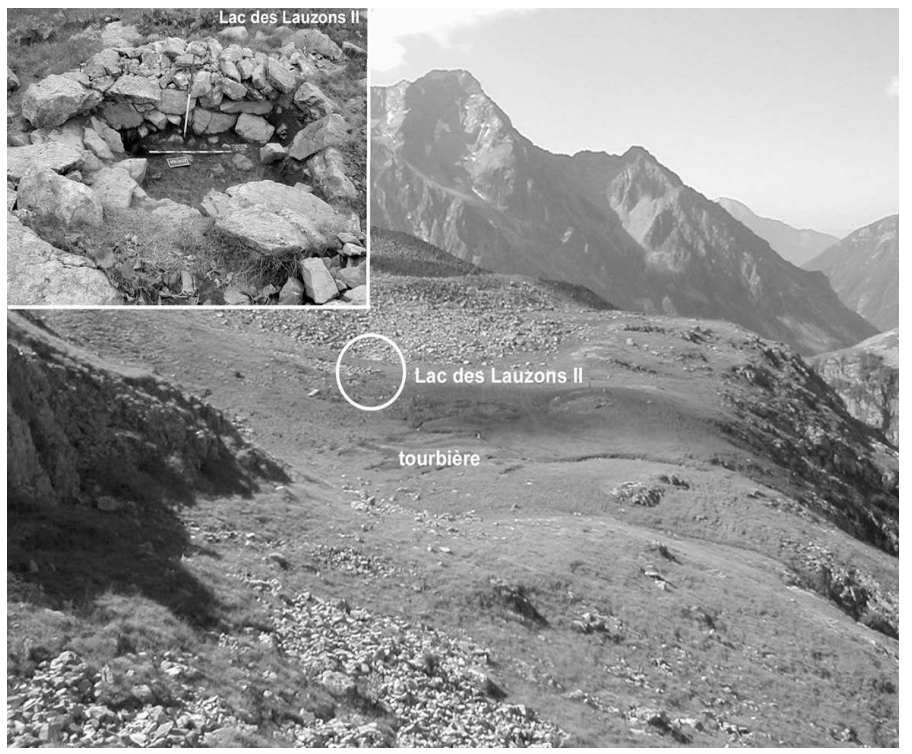

11 Vue depuis l'ouest du site du Lac des Lauzons II et de la cabane de l'âge du Bronze en cours de fouille à $2190 \mathrm{~m}$ d'altitude (Champoléon). Cliché M. Segard, juillet 2000. 
À l'image du site du Lac des Lauzons II, l'enclos de Faravel VIIId (fig. 2-12-13) avait été identifié comme un enclos lié à des cabanes médiévales situées quelques mètres plus en amont (Walsh 1999). La fouille entreprise en 1999 a révélé une structure E1 de forme ovale et de direction sud-ouest/ nord-est, mesurant 7,50 m de longueur sur 2,4 m dans la partie médiane, et $0,80 \mathrm{~m}$ de largeur environ aux extrémités. Isolée dans une petite dépression, elle est constituée, à l'ouest, par les murs M1-M2, séparés par un possible seuil de $2 \mathrm{~m}$ de large, et à l'est, par le mur M3 en grande partie détruit (important éboulis). Accolé à M1, un mur en forme d'ellipse (M4) semble délimiter un espace extérieur. Un sondage de $2 \mathrm{~m}$ x $5 \mathrm{~m}$, implanté depuis le parement externe ouest et sud du mur M1 jusqu'à l'intérieur de l'enclos, avait donc pour objectif d'appréhender la nature et la datation

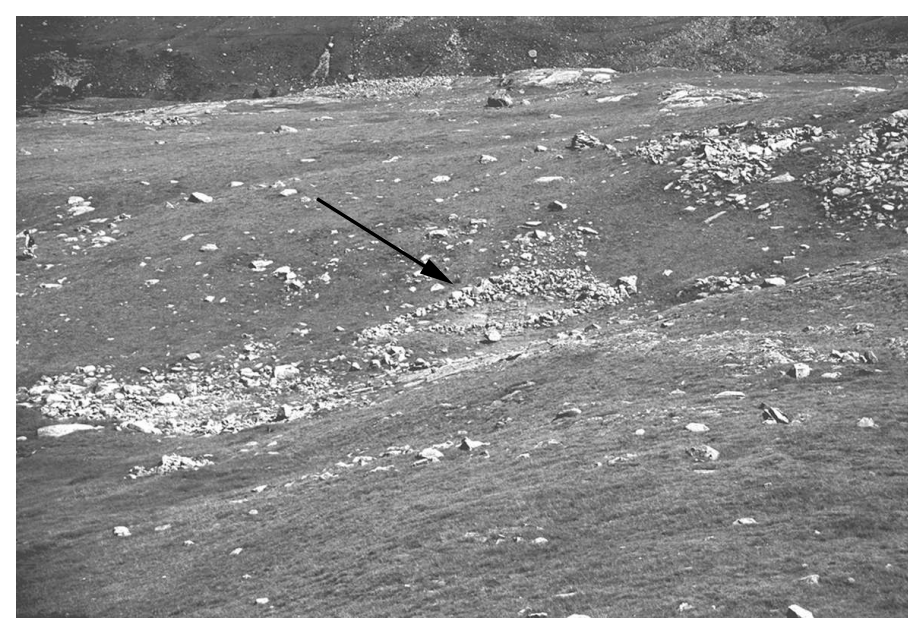

12 Vue depuis l'ouest de l'enclos pastoral de l'âge du Bronze du site de Faravel VIIId à 2165 m d'altitude (Freissinières). Cliché Fl. Mocci, août 1999.

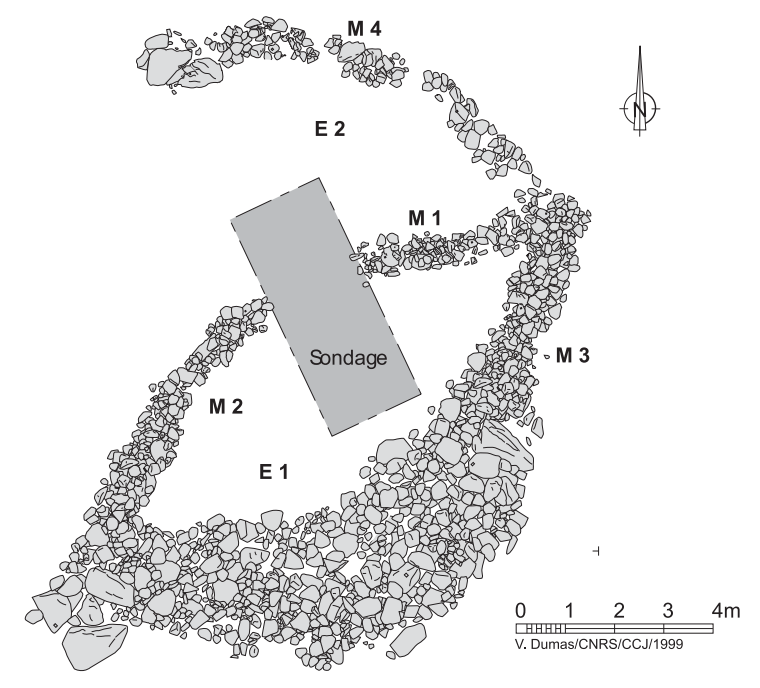

I 13 Relevé pierre à pierre des structures de l'enclos de Faravel VIIId (Freissinières). réelles de l'occupation. Les sept unités stratigraphiques identifiées témoignent essentiellement de phases de sédimentation interne au site et de phénomènes glaciaires antérieurs à l'occupation, elles révèlent que cette structure a été construite en tirant bénéfice de la topographie naturelle du secteur: utilisation d'une ravine de direction nordouest/sud-est contenant des amas de blocs afin d'asseoir les murs M1-M2; emploi des éboulis en place. Le seul objet mobilier consiste en une lamelle en silex dont on ne peut attester qu'elle soit contemporaine du site.

La datation ${ }^{14} \mathrm{C}$ des charbons recueillis dans l'enclos rattacherait l'occupation à l'âge du Bronze ancien, soit une fourchette chronologique comprise entre 2150 et 1920 av. J.-C. (3665 \pm 40 BP; Pa 1841). L'analyse anthracologique effectuée par B. Talon souligne la présence des taxons suivants: Picea/Larix (cf. Larix), Pinus cembra, Juniperus sp, Betula sp, écorce ou écaille de cône. La présence du pin cembro dans les assemblages anthracologiques confirme que cette essence a connu par le passé une extension insoupçonnée. La strate sous-ligneuse représentée par le genévrier ainsi que la présence d'espèces pionnières comme le mélèze et le bouleau illustrent la faible densité du couvert forestier. La présence de charbons de bois dispersés dans des niveaux de colluvionnement confirme l'origine in situ du bois carbonisé : cette végétation ouverte occupait les environs immédiats du site. Après l'incendie, naturel ou non, ces charbons ont été entraînés le long du versant par colluvionnement et solifluxion. En revanche, les charbons retrouvés dans le niveau d'occupation sont probablement le résultat d'une récolte du bois, mais à proximité du site, sans transport important de la part des humains, car ils n'apportent aucune modification dans la composition du peuplement végétal (Talon 2000, 41-43).

\section{Palynologie et dynamiques agro- sylvo-pastorales en milieu montagnard}

I objectif des analyses paléoenvironnementales est d'étudier, pour la période charnière de l'âge du Bronze, un petit terroir en haute résolution spatiale et temporelle dans le but d'aboutir à une reconstitution des modalités et chronologies de l'occupation de la montagne et de l'exploitation des terres à l'échelle de la vallée. La restitution des rythmes agropastoraux est fondée sur l'analyse pollinique de six séquences paléolacustres et tourbeuses holocènes prélevées sur l'ensemble de la vallée du Champsaur selon un transect altitudinal de 800 à $2200 \mathrm{~m}$, qui traverse différentes structures de végétation (fig. 14, tabl. I). L'échelle micro-régionale a été choisie afin de mieux évaluer le degré d'hétérogénéité spatiale de la gestion du territoire montagnard par les sociétés alpines à la lumière de sa dimension verticale. 


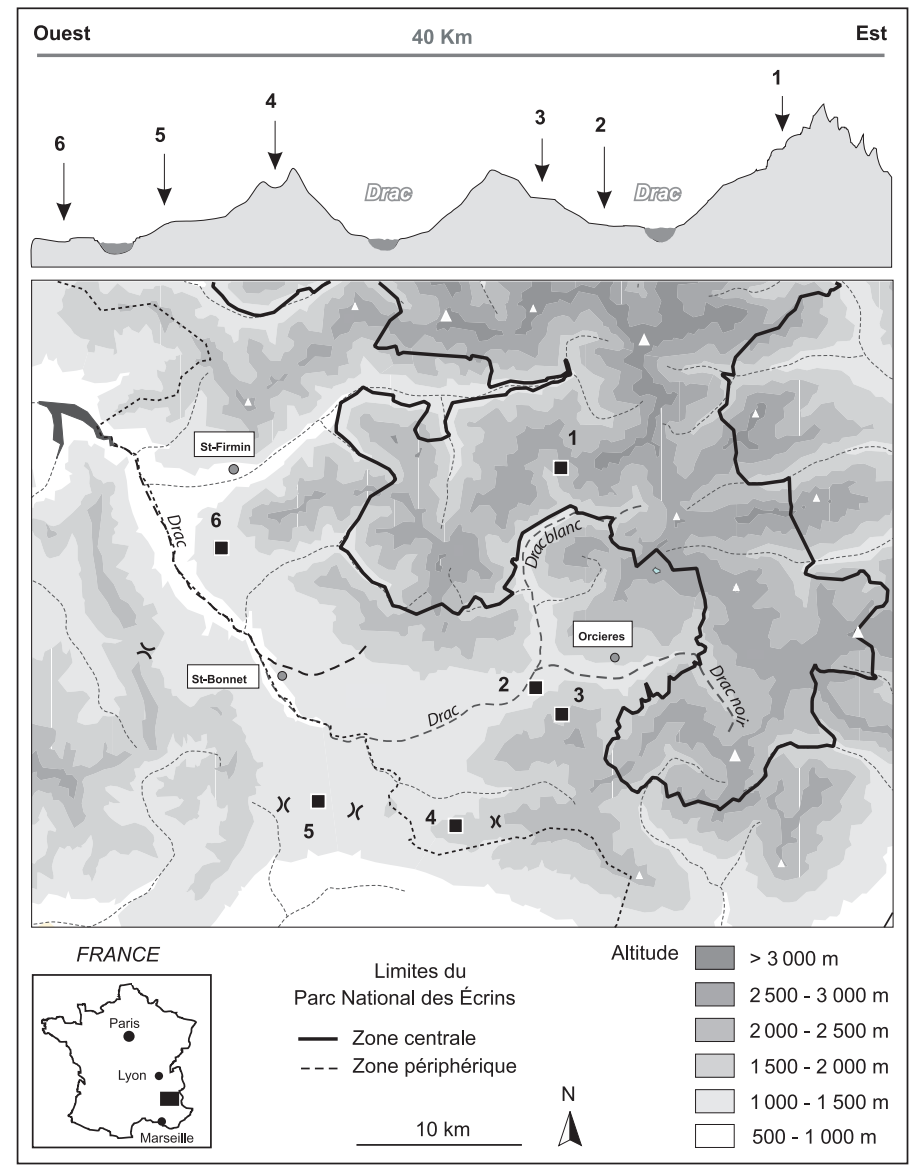

14 Localisation des six sites sondés pour analyses palynologiques dans la vallée du Champsaur (Alpes françaises du Sud). La description détaillée de chacun des sites est donnée dans le tableau I (M. Court-Picon, 2003).

La portée chronologique des séquences utilisées (à l'exception du site du Lac de Faudon - site 4 -) permet de suivre les grandes étapes de l'anthropisation de cette région au cours des dix derniers millénaires (fig. 15a-b, tabl. II).

Les premiers indices polliniques d'anthropisation apparaissent régionalement à partir du Néolithique ancien (5700-4500 av. J.-C.) attestant une fréquentation précoce de l'ensemble du milieu montagnard, y compris les zones de haute altitude aujourd'hui supra-forestières. Durant cette période, les signaux d'activité anthropique paraissent plus marqués dans les secteurs de bas plateaux et vastes replats, plus favorables en terme de ressources herbagères (Sagne de Canne - site 5 - $1272 \mathrm{~m}$, Tourbière de Libouse - site 3 $1455 \mathrm{~m}$ ), alors que seuls des indices très discrets d'activités agro-pastorales ou de déboisements ponctuels caractérisent l'anthropisation des zones de haute altitude et de fond de vallée en bordure du Drac (Lac des Lauzons - site 1 2180 m, Tourbière du Lauza - site 2 - 1140 m, Étang du Laux du Villardon - site 6 - $1090 \mathrm{~m}$ ). Au cours des périodes du Néolithique moyen (4500-3500 av. J.-C.) et Néolithique final (3500-2800 av. J.-C.), les zones hautes semblent avoir été occupées de manière durable avec vraisemblablement des fréquentations saisonnières et la mise en place d'estivages. À plus basse altitude, ces phases se caractérisent par des emprises bien marquées témoins d'une certaine mobilité des pratiques, mais qui, en terme de pression anthropique, n'apparaissent pas plus affirmées.

C'est à partir de l'âge du Bronze que les données pollenanalytiques indiquent une intensification des déboisements à toutes les altitudes provoquant une descente de la limite supérieure des forêts et une ouverture du milieu alors nécessaire à la constitution des terroirs agricoles et pastoraux (Beaulieu et al. 1990; Édouard et al. 1991; Ponel et al. 1992; Tessier et al. 1993; Talon et al. 1998; Thinon, Talon 1998). Enregistrées dans la quasi-totalité des séquences polliniques de la vallée, les emprises de cette période constituent ainsi la première phase d'expansion majeure des activités humaines, et semblent révéler un phénomène à caractère régional.

Les deux phases d'ouverture des sapinières d'altitude mises en évidence dans l'enregistrement pollinique des zones hautes au Bronze ancien puis au Bronze final (Lac des Lauzons - site 1 - $2180 \mathrm{~m}$ ), peuvent être interprétées comme un abaissement significatif de la limite supérieure des forêts subalpines et/ou la création de voies de passage sur le versant pour la conduite de troupeaux plus importants à l'estive. Elles sont associées à une augmentation de la représentation des marqueurs polliniques de plantes rudérales et pastorales (armoises, plantains, rumex), et à la présence de spores de champignons coprophiles appartenant à la famille des Sordariacées (Neurospora sp., Cercophora, Sporormiella) se développant sur les déjections animales, toutes deux liées à la constitution des alpages supra-forestiers

\begin{tabular}{c|c|cccc}
\hline $\mathrm{N}^{\circ}$ & Nom & Description du site - exposition & Altitude & Latitude & Longitude \\
\hline 1 & Lac des Lauzons & petite tourbière au cœur d'un espace pastoral de haute altitude - $\mathrm{SO}$ & $2180 \mathrm{~m}$ & $44^{\circ} 47^{\prime} 01^{\prime \prime} \mathrm{N}$ & $6^{\circ} 17^{\prime} 19^{\prime \prime} \mathrm{E}$ \\
\hline 2 & Le Lauza & tourbière située au sein d'un paysage agropastoral bocager - BV & $1140 \mathrm{~m}$ & $44^{\circ} 39^{\prime} 22^{\prime \prime} \mathrm{N}$ & $6^{\circ} 12^{\prime} 39^{\prime \prime} \mathrm{E}$ \\
\hdashline 3 & Libouse & tourbière sur un replat herbagé pâturé en ubac forestier (mélézin) - N & $1455 \mathrm{~m}$ & $44^{\circ} 38^{\prime} 01^{\prime \prime} \mathrm{N}$ & $6^{\circ} 13^{\prime 2} 25^{\prime \prime} \mathrm{E}$ \\
\hline 4 & Lac de Faudon & petit lac près d'un col, en zone pastorale d'altitude d'été - SO & $1577 \mathrm{~m}$ & $44^{\circ} 36^{\prime} 10^{\prime \prime} \mathrm{N}$ & $6^{\circ} 12^{\prime} 58^{\prime \prime} \mathrm{E}$ \\
\hdashline 5 & Sagne de Canne & tourbière sur un vaste plateau ouvert exploité en prairies de fauche - $\mathrm{P}$ & $1272 \mathrm{~m}$ & $44^{\circ} 37^{\prime} 05^{\prime \prime} \mathrm{N}$ & $6^{\circ} 06^{\prime} 06^{\prime \prime} \mathrm{E}$ \\
\hline 6 & Le Laux du Villardon & petit étang ourlé de haies et au cour d'un secteur agricole (maïs) - BV & $1090 \mathrm{~m}$ & $44^{\circ} 43^{\prime} 31^{\prime \prime} \mathrm{N}$ & $6^{\circ} 02^{\prime} 52^{\prime \prime} \mathrm{E}$ \\
\hline
\end{tabular}

Tableau I - Description des six sites de la vallée du Champsaur (Alpes françaises du Sud) explorés pour sondages et analyses palynologiques (M. Court-Picon). SO : exposition sud-ouest ; BV : position en bas de versant, en zone de plaine alluviale ; N : exposition nord ; P : zone plane. 

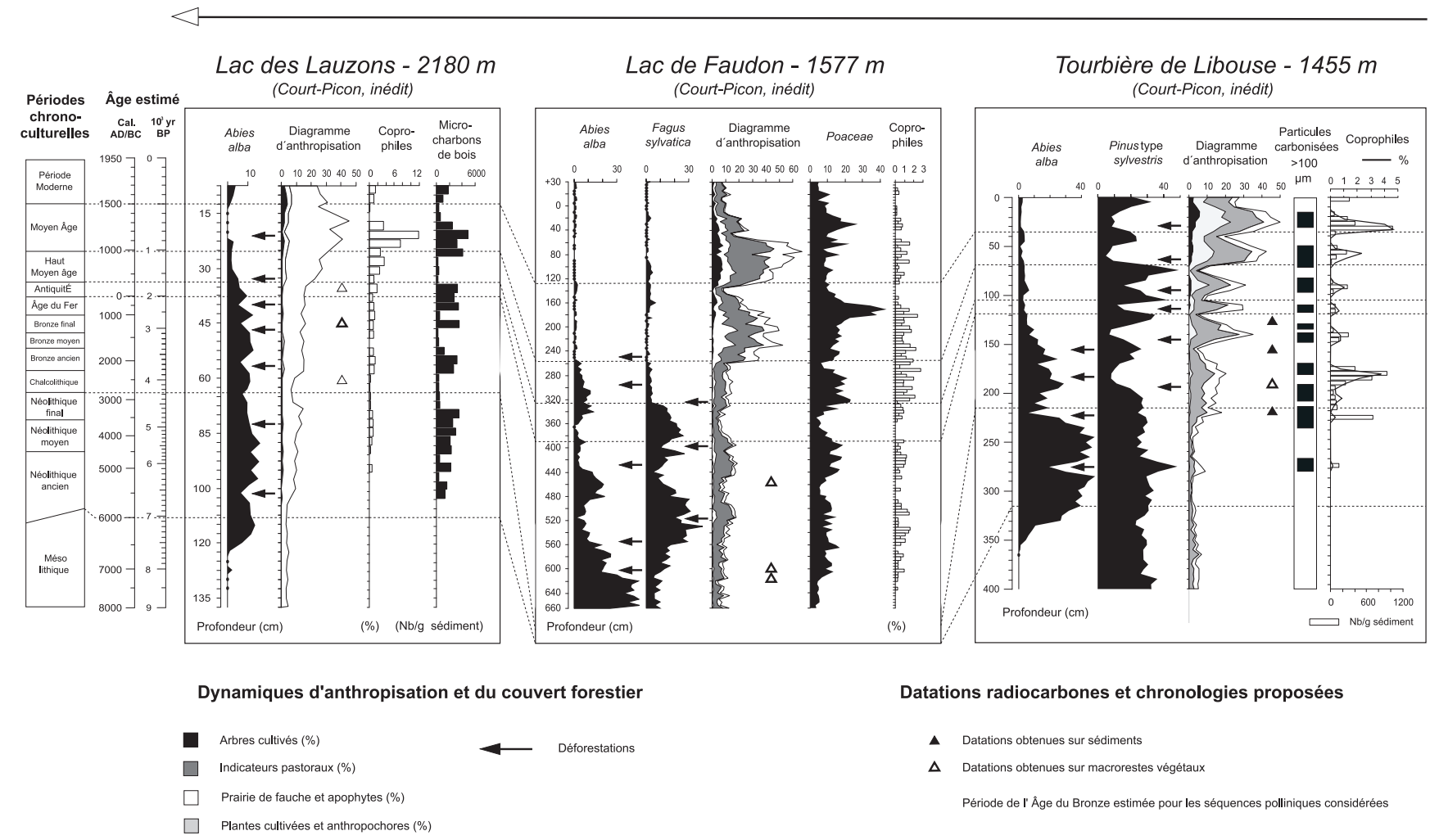

Datations radiocarbones et chronologies proposées

Milieux montagnards

Zones de fond de vallée

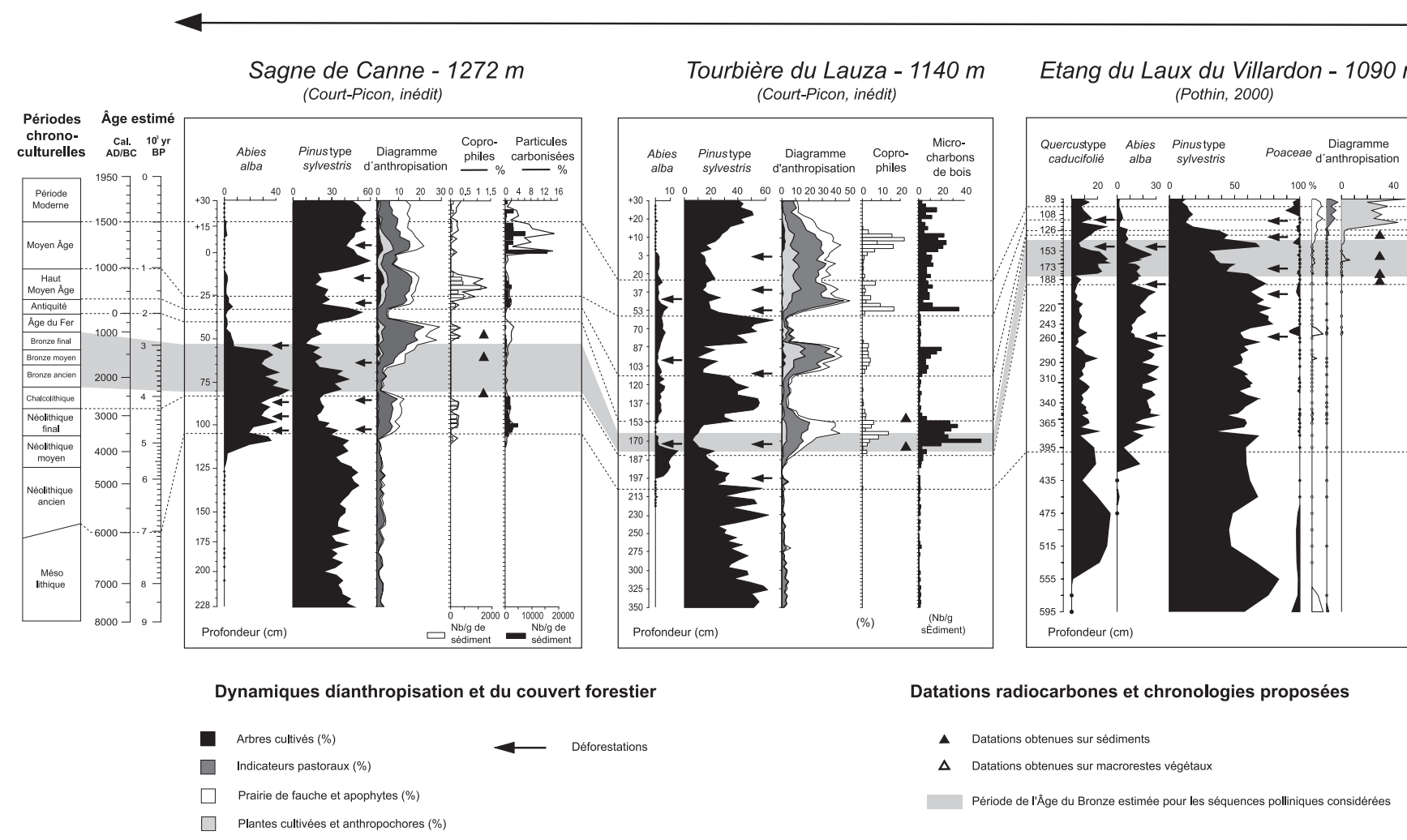

I 15a-b Palynologie et histoire des activités humaines en milieu montagnard dans la vallée du Champsaur (Alpes françaises du Sud) au cours de l'Holocène: évolution comparée de la végétation et du signal incendie établie à partir de la variation des indices polliniques d'anthropisation et autres éléments du palynofaciès (micro-fossiles non polliniques et micro-charbons de bois) (M. Court-Picon, 2003). 


\begin{tabular}{|c|c|c|c|c|c|c|}
\hline $\mathrm{N}^{\circ}$ & Nom & Niveaux $(\mathrm{cm})$ & Évènement paléobotanique & Matériel & Age uncal. BP & Date calibrée ( 2 sigmas) \\
\hline 1 & Lac des Lauzons & $34-35$ & déboisements massifs et forte fréquentation pastorale & gyttja & $1947 \pm 46$ & 36 av. J.-C. -145 ap. J.-C. \\
\hline 1 & Lac des Lauzons & $44-45$ & attaques localisées des sapinières sur les versants & bois Betula sp. & $2949 \pm 53$ & 1312 - 994 av. J.-C. \\
\hline 1 & Lac des Lauzons & $60-61$ & nouvelle occupation de l'espace pastoral & gyttja & $4136 \pm 52$ & $2882-2500$ av. J.-C. \\
\hline 2 & Le Lauza & $152-153$ & déclin des pratiques agro-pastorales & tourbe & $2021 \pm 62$ & 179 av. J.-C. - 122 ap. J.-C. \\
\hline 2 & Le Lauza & $172-173$ & déboisements et mise en valeur des terres & tourbe & $3018 \pm 38$ & $1391-1123$ av. J.-C. \\
\hline 3 & Libouse & $124-125$ & recul des activités humaines & tourbe & $2154 \pm 64$ & $374-43$ av. J.-C. \\
\hline 3 & Libouse & 154-155 & nouvelle emprise agro-pastorale & tourbe & $2534 \pm 44$ & $802-514$ av. J.-C. \\
\hline 3 & Libouse & $189-190$ & phase d'ouverture des pinèdes & bois Abies alba & $3189 \pm 35$ & $1519-1398$ av. J.-C. \\
\hline 3 & Libouse & $219-220$ & ouverture des sapinières, premières cultures & tourbe & $4421 \pm 37$ & $3297-2918$ av. J.-C. \\
\hline 4 & Lac de Faudon & 454-455 & reprise des sapinières, milieu fermé & bois Abies alba & $2350 \pm 34$ & $414-376$ av. J.-C. \\
\hline 4 & Lac de Faudon & $599-600$ & phase de déforestation sélective sur le sapin & bois Abies alba & $2875 \pm 39$ & $1157-918$ av. J.-C. \\
\hline 4 & Lac de Faudon & $619-620$ & environnement forestier (sapinière) & bois Abies alba & $3004 \pm 47$ & $1392-1058$ av. J.-C. \\
\hline 5 & Sagne de Canne & $46-47$ & intensification des activités humaines & tourbe & $2179 \pm 35$ & $365-110$ av. J.-C. \\
\hline 5 & Sagne de Canne & $59-60$ & début d'une nouvelle phase d'anthropisation & tourbe & $3378 \pm 37$ & $1743-1523$ av. J.-C. \\
\hline 5 & Sagne de Canne & $79-80$ & diminution des indicateurs agro-pastoraux & tourbe & $3996 \pm 38$ & $2584-2408$ av. J.-C. \\
\hline 6 & Le Laux du Villardon & $127-130$ & phase d'intensification des activités anthropiques & tourbe & $2115 \pm 70$ & $367-57$ ap. J.-C. \\
\hline 6 & Le Laux du Villardon & $158-160$ & brève reprise des sapinières, maintien des activités & tourbe & $3740 \pm 60$ & $2318-1949$ av. J.-C. \\
\hline 6 & Le Laux du Villardon & $179-181$ & déboisement des pinèdes, intensification agraire & tourbe & $3930 \pm 50$ & $2564-2210$ av. J.-C. \\
\hline 6 & Le Laux du Villardon & $181-184$ & ouverture des sapinières et favorisation des chênaies & tourbe & $4175 \pm 45$ & $2887-2588$ av. J.-C. \\
\hline
\end{tabular}

Tableau II - Tableau récapitulatif des datations radiocarbones $\left({ }^{14} \mathrm{C}\right)$ obtenues sur les sédiments et macrorestes végétaux des six sondages palynologiques dans la vallée du Champsaur (Alpes françaises du Sud, France) (M. Court-Picon).

locaux. Le fait que ces marqueurs ne fléchissent pas au cours des périodes du Bronze moyen et Bronze final semble plaider pour une occupation du site sur la longue durée et confirme ainsi les indices archéologiques de croissance continue suggérés par les structures d'habitats datées de cette période et présentes à proximité (Palet et al. 2003).

Plus bas en altitude, la période de l'âge du Bronze se caractérise par une diversification des activités anthropiques et une hétérogénéité spatiale des pratiques. L'enregistrement pollinique du petit Lac de Faudon, à $1577 \mathrm{~m}$ d'altitude (site 4), qui débute au cours du Bronze moyen, présente des indices très importants d'exploitation forestière touchant les forêts montagnardes. Après une phase de faible pression humaine sur le milieu dominé par les sapinières, le Bronze final se caractérise par un déboisement sélectif sur le sapin qui par le jeu de la concurrence favorise le développement du hêtre (Nakagawa 1998). Les raisons de ce déboisement peuvent être multiples : utilisation du bois d'œuvre du sapin pour la construction d'habitat (Küster 1994; Nakagawa et al. 2000) ou pour l'alimentation des forges qui ont été identifiées au sein d'un gisement archéologique découvert à proximité et présentant une phase d'occupation à l'âge du Bronze (Beaulieu et al. 2003), ou simplement pour favoriser la hêtraie à vocation sylvo-pastorale (Björkman 1997). Dans tous les cas ces défrichements ne sont pas ponctuels et s'inscrivent dans la longue durée puisqu'ils se poursuivront au cours de l'âge du Fer.

Les déforestations similaires observées dans d'autres secteurs du Champsaur ne connaissent pas systématiquement la même finalité. La sapinière très dense qui colonisait alors le versant de la Tourbière de Libouse (- site 3 - 1455 m) va être intensément défrichée dès le Néolithique final pour une exploitation pastorale du plateau et l'initiation des premières cultures céréalières. Au Bronze ancien, cette emprise agropastorale va s'intensifier et s'étendre avec l'appropriation des terres jusque-là occupée par les pinèdes et localement sur la tourbière elle-même avec le défrichement de l'aulnaie locale, emprise qui restera très forte tout au long de l'âge du Bronze jusqu'au premier âge du Fer qui enregistre une déprise.

Aujourd'hui largement exploité pour la production de fourrage, le secteur très ouvert de Sagne de Canne (- site 5 - 1272 m) connait, à l'âge du Bronze, une phase de déprise (Bronze ancien) à la suite d'une forte occupation Néolithique avec une reprise des pinèdes, des feuillus mésophiles, dans une moindre mesure des sapinières, et le développement local d'une aulnaie dense. Une nouvelle ouverture du milieu se manifeste dès le début du Bronze moyen sous l'impulsion d'une expansion de pratiques diversifiées et la mise en place de systèmes mixtes agro-sylvo-pastoraux (expansion des indicateurs d'activités agricoles et pastorales et développement du hêtre et du chêne favorable à la fréquentation des troupeaux). Cette reprise se poursuivra et s'intensifiera au cours des périodes du Bronze final, de l'âge du Fer et du début de l'époque romaine.

Bien que l'expansion de l'utilisation des terres et l'accentuation des déforestations à l'âge du Bronze s'observent à toutes les altitudes, c'est dans les secteurs de fond de vallée que la pression anthropique sur le milieu est la plus forte mais aussi la plus pérenne. L'enregistrement pollinique de 
la Tourbière du Lauza, (- site 2 - $1140 \mathrm{~m}$ ) présente tout au long de cette période des défrichements importants sur l'ensemble des formations forestières qui couvraient alors le secteur; sapinières, pinèdes, mais aussi chênaies mixtes sont défrichées principalement pour une utilisation pastorale du sol; on note une explosion des Poaceae, des indicateurs pastoraux et de l'ensemble des autres apophytes, liées aux activités humaines, en parallèle avec l'apparition de spores de champignons coprophiles, en relation avec la présence locale des troupeaux, et de champignons carbonicoles associés à la forte concentration en particules carbonisées observée, témoin de l'utilisation du feu pastoral pour les défrichements et la mise en valeur des terres (Vannière 2001; Vannière et al. 2001 ; Galop et al. 2003).

L'étude palynologique du site de l'étang du Laux du Villardon (- site 6-1090 m), situé sur une terrasse glacio-lacustre de fond de vallée, dans un secteur aujourd'hui largement dévolu aux cultures céréalières et fourragères, enregistre également à l'âge du Bronze une forte emprise agropastorale, dans la continuité de la reprise observée dès le début du Chalcolithique (Pothin 2000). D'intenses attaques sur les peuplements de sapins puis de pins qui dominaient alors le paysage du Néolithique sont opérées pour ouvrir des espaces à vocation principalement sylvo-pastorale avec notamment la favorisation des chênaies propices au pacage des troupeaux (feuillage, glands).

\section{Pastoralisme et structures bâties au-dessus de $2000 \mathrm{~m}$ au cours de l'âge du Bronze}

$\mathrm{L}^{\prime}$ ' ensemble de ces nouveaux travaux archéologiques et paléoenvironnementaux alpins démontre que la fin de la Préhistoire, entre le Néolithique final et l'âge du Bronze, est marquée par un développement du peuplement et des modifications importantes et durables dans le paysage. Cet événement n'est pas un phénomène local ou régional: il correspond à des changements qui interviennent dans l'ensemble de l'arc alpin mais aussi dans les montagnes pyrénéennes (Galop 1998; Galop et al. 2003; Vannière et al. 2001; Rendu et al. 1995). Dans les Alpes Suisses occidentales, un programme de recherche très développé a démontré que les étages subalpins et alpins ont été défrichés et exploités en tant que pâturage dès le Néolithique, avec une intensification d'activité au cours de l'âge du Bronze caractérisée par l'exploitation de mines de cuivre (Curdy et al. 1999). Il est clair que la fin du Néolithique et le début de l'âge du Bronze (mais probablement antérieurement dans certaines micro-régions alpines) correspondent à une première phase d'expansion qualifiée de «vraie phase d'exploration des hauteurs » dans laquelle on peut voir plusieurs origines (Primas 1992): recherche et exploitation de minerais,
« aspect religieux » de la montagne (Lumley 1995), structuration de l'espace par l'art rupestre (Frachetti, Chippindale 2002) mais aussi activités pastorales. Dans la Vallée des Merveilles par exemple, la présence de troupeaux a été clairement identifiée par la palynologie (Kharbouch, Gauthier 2000). Sur les hauts massifs du Champsaur et de Freissinières, l'archéologie témoigne de l'existence de structures pastorales bâties et de l'augmentation des activités dès la fin du Néolithique-début de l'âge du Bronze. Antérieurement à cette période, nous pouvons nous interroger sur l'absence de structures ou d'aménagements en pierre alors que les premiers indices polliniques d'anthropisation apparaissent à partir du Néolithique ancien (cf. supra $\S 4$ ). Sans doute faut-il envisager des sites en abris sous roche, très peu fréquents sur notre zone d'étude, ou en matériaux périssables. La découverte, sur le plateau de Faravel, de trois trous de poteau du premier âge du Fer creusés sur un petit replat déjà occupé au Mésolithique ancien atteste la présence de ce type d'aménagement à plus de $2200 \mathrm{~m}$ d'altitude (Faravel XIII, fig. 2; Walsh 2001; Walsh, Mocci 2003a).

La présence de structures bâties de l'âge du Bronze situées au-dessus de $2000 \mathrm{~m}$ semble relativement rare ou est peu évoquée dans l'arc alpin. Même si nombre de chercheurs soutiennent l'hypothèse d'une augmentation de l'activité humaine dans les zones d'altitude au cours de l'âge du Bronze, très peu font état de constructions ou d'aménagements. Nous prendrons pour exemple, les travaux menés dans la région de Trentino-Alto qui révèlent une augmentation $\mathrm{du}$ nombre de sites en altitude, l'altitude moyenne d'installation passant de $500 \mathrm{~m}$ à la fin du Néolithique à $850 \mathrm{~m}$ au cours de l'âge du Bronze, sans préciser toutefois la nature et la fonction de ces sites (Di Pillo 1999). Dans la Val Venosta, mention est faite d'un site "domestique" du début de l'âge du Bronze à 1049 m d'altitude (Kaufmann, Nothdurfter 1997). Un des sites les plus élevés en altitude en France est celui du Coin à Aussois (Haute-Maurienne) implanté à $1500 \mathrm{~m}$. Plus de 700 fragments de céramiques non tournées de l'âge du Bronze moyen et du Bronze final ont été recueillis mais aucun vestige de construction n'est mentionné. L'un des objectifs des travaux entrepris sur le site du Coin est d'aborder la question des relations «bipolaires entre les versants ouest et est des Alpes entre la fin du Bronze moyen et le début du Bronze final à partir d'une analyse des documents céramiques» (Ozanne, Vital 1999, 520). L'absence de marqueurs chronologiques tels que le mobilier céramique ou métallique sur l'ensemble de nos sites fouillés peut être certes liée à des problèmes de taphonomie ou d'acidité des sols mais aussi au caractère marginal de l'occupation humaine, saisonnière et temporaire, sur ces zones d'altitude.

Les résultats des analyses paléoenvironnementales entreprises dans le cadre de nos travaux pluridisciplinaires sur la 
montagne sud-alpine apparaissent en parfait accord avec les nouvelles données archéologiques obtenues sur la haute montagne champsaurine, sur le plateau et la Montagne de Faravel et sur la haute vallée de Chichin. Elles semblent, en revanche, ne pas refléter les fluctuations globales du peuplement enregistrées en haute montagne par les anciennes études archéologiques réalisées à l'échelle de l'arc alpin, qui étaient expliquées par le jeu des améliorations et péjorations climatiques. C'est particulièrement le cas pour le Bronze moyen, où la raréfaction des sites archéologiques a souvent été expliquée par la péjoration climatique qui atteint l'Europe aux $\mathrm{XVII}^{\mathrm{e}}-\mathrm{XV}^{\mathrm{e}} \mathrm{s}$. avant notre ère. (Segard et al. 2002). Dans ce contexte, les apports de la palynologie et des nouvelles méthodes de l'archéologie du paysage permettent de montrer que la péjoration climatique du Bronze moyen ne semble pas avoir impliqué un mouvement massif de descente des populations humaines vers le fond de vallée, une continuité dans l'occupation de la haute montagne étant observée tout au long de l'âge du Bronze. La période de l'âge du Bronze apparaît ainsi comme un seuil dans l'histoire de l'anthropisation de la montagne champsaurine, en particulier en ce qui concerne le développement des pratiques agraires dans les zones de basse et moyenne altitude. De légers asynchronismes quant à l'intensification des activités humaines qui se manifeste à l'âge du Bronze sont néanmoins observés par la palynologie entre les différents sites intra-valléens, avec pour certains des déboisements importants opérés dès le Néolithique final et le Bronze ancien, alors que d'autres secteurs semblent avoir été exploités intensivement plus tardivement à partir du Bronze moyen ou seulement au Bronze final (fig. 15a-b, tabl. II). Cependant, aucune relation n'est observée entre ces discordances et des paramètres physiques tels que l'altitude, la topographie, l'exposition ou les conditions d'accès, qui auraient pu expliquer une occupation plus tardive, à la faveur d'amélioration climatique et/ou d'innovations technologiques. Si le début de l'âge du Bronze se caractérise par une fréquentation plus importante de la montagne qui serait véritablement mise en valeur à partir de cette période, il n'y a jamais de désertification totale du domaine montagnard tout au long de l'âge du Bronze, qu'il s'agisse de sites de moyenne ou de très haute altitude. Il semble, à la lumière des résultats présentés ici, que si le Bronze final constitue partout la phase la plus intensive de l'exploitation agropastorale de la vallée, la période du Bronze moyen se caractérise plutôt par des mouvements de population à l'échelle de la vallée avec le déplacement de groupes humains sur quelques kilomètres pour mettre en valeur de nouvelles terres - pratiques agro-pastorales relativement mobiles ou système agro-forestier (Galop et al. 2003) - plutôt que par de réelles fluctuations de la démographie. Les populations alpines de l'âge du Bronze qui fréquentaient le Haut Champsaur et la vallée de Freissinières à cette période semblent ainsi bien adaptées aux conditions du domaine montagnard, l'occupation de la haute montagne n'étant pas étroitement corrélée aux fluctuations climatiques qui ont

Notes de commentaire

affecté la vallée à l’âge du Bronze.

*Kevin WALSH - Department of Archaeology, University of York, King's Manor - YOEP17 York, Grande Bretagne.

**Florence Mocci - Centre Camille Jullian, UMR 6573 CNRS-Université de Provence, Maison Méditerranéenne des Sciences de l'Homme, 5 rue du Château de l'Horloge, BP 647 - 13094 Aix-en-Provence cedex 2.

***Mona COURT-Picon - Institut Méditerranéen d'Écologie et de Paléoécologie, CNRS 6116, Université Aix-Marseille III, Europôle de l'Arbois, BP 80 13545 Aix-en-Provence cedex 4.

****Stefan TzorTzIs - Service Archéologie, ville de Martigues, Hôtel de ville BP 101 - 13692 Martigues cedex et UMR 6578 CNRS-Université de la Méditerranée, Faculté de Médecine, 27 bd Jean Moulin - 13385 Marseille cedex 5.

*****Josep-Maria PALET-MartinEZ - Institut Catala d'Arqueologia Classica, Plaça Rovellat, s/n 43003 - Tarragona, Espagne.

Vincent Dumas - Centre Camille Jullian, UMR 6573 CNRS-Université de Provence, Maison Méditerranéenne des Sciences de 1'Homme, 5 rue du Château de l'Horloge, BP 647 - 13094 Aix-en-Provence cedex 2.

Vanessa Py - Laboratoire d'Archéologie Méditerranéenne Médiévale, CNRSUniversité de Provence, Maison Méditerranéenne des Sciences de l'Homme, 5 rue du Château de l'Horloge, BP 647 - 13094 Aix-en-Provence cedex 2.

Maxence Segard - Centre Camille Jullian, UMR 6573 CNRS-Université de Provence, Maison Méditerranéenne des Sciences de l'Homme, 5 rue du Château de l'Horloge, BP 647 - 13094 Aix-en-Provence cedex 2.

Brigitte TALON - Institut Méditerranéen d'Écologie et de Paléoécologie, CNRS 6116, Université Aix-Marseille III, Europôle de l'Arbois, BP 80 - 13545 Aix-
en-Provence.

1 Programme Collectif de Recherche "Occupation du sol et pastoralisme de la Préhistoire au Moyen Âge dans le sud du massif alpin” (coord. Ph. Leveau, Centre Camille Jullian); Programme Environnement, Vie et Sociétés du CNRS "La forêt et le troupeau dans les Alpes du sud (Hautes-Alpes) du tardiglaciaire à l'époque actuelle à l'interface des dynamiques naturelles et des dynamiques sociales" (coord. Ph. Leveau et J.-L. de Beaulieu); Thème de Recherche $\mathrm{n}^{\circ} 5 \mathrm{du}$ Centre Camille Jullian "Territoires, Peuplement et Réseaux".

2 L'ensemble des opérations archéologiques est conduit sous la direction scientifique de Florence Mocci, de Kevin Walsh et de Josep Palet-Martinez avec la collaboration de Vincent Dumas et Maxence Segard, de François Ricou (Communautés des Communes du Haut Champsaur) et de bénévoles ayant une connaissance du milieu alpin. Elles ont bénéficié de la collaboration scientifique et financière du Ministère de la Culture, du Parc National des Écrins, des Communautés des Communes du Pays des Écrins et du Canton du Haut Champsaur, du Centre Camille Jullian (UMR 6573 CNRS-Université de Provence), de l'Université de York, de la British Academy et du DURSI de la Generalitat de Catalunya.

3 Lac des Lauzons sur la commune de Champoléon et Abri Fangeas sur la commune de Freissinières (données analysées par Jacques-Louis de Beaulieu et Mona Court-Picon

4 Ces analyses ont été réalisées par Aline Durand (Laboratoire d'Archéologie Méditerranéenne Médiévale, CNRS UMR 6572), Brigitte Talon et Vanessa Py.

5 Analyses conduites par K. Walsh. 
6 Savoie, Chartreuse, Vercors, Dévoluy, Büech et Laragnais.

7 Ces analyses ont réalisées par J.-F. Saliège (UMR 121, Laboratoire d'Océanographie Dynamique et Climatologique de Jussieu (Pa...) et par T. Goslar (Pozna Radiocarbon Laboratory, Foundation of the Adam Mickiewicz University, Pologne (Pz...). Les datations proposées pour les sites de notre secteur d'étude ont été calibrées avec un indice de confiance de probabilité de deux sigma $(95,4 \%$ de confiance).

8 Analyses réalisées par V. Py.

9 Analyses réalisées par M. Court-Picon.

10 Des prélèvements de ce niveau d'occupation ont été recueillis et font l'objet d'analyses palynologiques, sédimentologiques et pédologiques en cours actuellement par J.-L. de Beaulieu (IMEP, CNRS) et K. Walsh.

Barge et al. 1998 : BARGE (H.), BOURHIS (J.-R.), ROSTAN (P.), GUENDON (J.-L.) - La mine des Clausis à Saint-Véran (Hautes-Alpes). Exploitation et aire de réduction du minerai de cuivre d'époque préhistorique. In: MORDANT (C.), PERNOT (M.), RYCHNER (V.) éd. - L'atelier du bronzier en Europe du XX $X^{e}$ au VIII ${ }^{e}$ s. avant notre ère. Tome II: Du minerai au métal, du métal à l'objet. Actes du colloque international Bronze 96, Neuchâtel/Dijon, 4-9 mars 1996. Paris, CTHS, 1998, pp. 71-81.

Beaulieu et al. 1990 : BEAULIEU (J.-L. de), ÉDOUARD (J.-L.), PONEL (Ph.), ROLANDO (C.), TESSIER (L.), THINON (M.), THOMAS (A.) - Timber line and human impact in the French Alps. The state of the art and research programs. In: Impact of Prehistoric and Medieval Man on the Vegetation: Man at the Forest Limit. Report of the meeting PACT network 31-10, Palaeoecology, Ravello, 1989. Pact Belgium, Rixensart, 1990, pp. 63-80.

Beaulieu et al. 2003 : BEAULIEU (J.-L. de), LEVEAU (Ph.), MIRAMONT (C.), PALET (J.-M.), WALSH (K.), COURT-PICON (M.), RICOU (F.), SEGARD (M.), SIVAN (O.), ANDRIEU-PONEL (V.), BADURA (M.), BERTUCCHI (G.), BOUTTERIN (C.), DURAND (A.), ÉDOUARD (J.-L.), LAVOIE (M.), MORIN (A.), MOCCI (F.), PONEL (Ph.), POTHIN (A.), PY (V.), TALON (B.), TZORTZIS (S.), BONET (R.), COLUMEAU (Ph.), CORTOT (H.), GARCIA (D.) - Changements environnementaux postglaciaires et action de l'homme dans le bassin de Buëch et en Champsaur (Hautes-Alpes, France). Premier bilan d'une étude pluridisciplinaire. In: Des milieux et des Hommes. E. SAS. Paris, 2003, pp. 79-87.

Bintz 1994 : BINTZ (P.) - La fin du Paléolithique et le Mésolithique dans les Alpes du Nord françaises: paléoenvironnement, peuplement et modes d'exploitation du milieu. Preistoria Alpina, 28, 1994, pp. 255-273.

Bintz 2004 : BINTZ (P.) - Mésolithique et Néolithique ancien. In : JOURDAINANNEQUIN (C.) dir. - Atlas culturel des alpes occidentales, De la Préhistoire à la fin du Moyen Âge, Programme ERICA. Paris, Picard, 2004, pp. 38-39.

Bintz, Bracco 2004 : BINTZ (P.), BRACCO (J.-P.) - Paléolithique supérieur récent. In: JOURDAIN-ANNEQUIN (C.) dir. - Atlas culturel des Alpes occidentales, De la Préhistoire à la fin du Moyen Âge, Programme ERICA. Paris, Picard, 2004, pp. 34-35.

Bintz, Morin 2001 : BINTZ (P.), MORIN (A.) - Dévoluy. Massifs et piémonts. Bilan Scientifique 2000. Service Régional de l'Archéologie PACA, Ministère de la Culture, 2001, p. 46.

Björkman 1997 : BJORKMAN (L.) - The role of human disturbance in the local Late Holocene establishment of Fagus and Picea forests at Flahult, western Smaland, southern Sweden. Vegetation History and Archaeobotany, 6, 1997, pp. 79-90.

Bocquet 2004a: BOCQUET (A.) - Bronze ancien, moyen et final. In: JOURDAIN-ANNEQUIN (C.) dir.: Atlas culturel des Alpes occidentales, De la Préhistoire à la fin du Moyen Âge, Programme ERICA. Paris, Picard, 2004, pp. 44-47.

Bocquet 2004b: BOCQUET (A.) - Néolithique moyen et final In: JOURDAINANNEQUIN (C.) dir. : Atlas culturel des Alpes occidentales, De la Préhistoire à la fin du Moyen Âge, Programme ERICA. Paris, Picard, 2004, pp. 40-41.

Bressy 2002 : BRESSY (C.) - Les matières premières des sites mésolithiques et néolithiques du plateau de Faravel (Freissinieres, 05). Étude de provenance du silex. In: WALSH (K.), MOCCI (Fl.) - Sondages archéologiques sur trois sites d'altitude de la Montagne de Faravel et de l'Abri Fangeas: Faravel XIX, Fangeas VI et Fangeas VII et prospection-inventaire dans la haute vallée de Chichin (05). Document Final de Synthèse, SRA PACA - Centre Camille Jullian - Parc national des Écrins, Aix-en-Provence, 2002, 137 p.

Brochier 2005 : BROCHIER (J.-E.) - Derniers chasseurs cueilleurs provençaux. In : DELESTRE (X.) dir. - Quinze d'archéologie en région PACA. Aix-enProvence, Édisud, 2005, pp. 26-31.

Brochier et al. 1999 : BROCHIER (J.-L.), BEECHING (A.), SIDI MAAMAR (H.), VITAL (J.) - Les grottes bergeries des Préalpes et le pastoralisme alpin, durant la fin de la Préhistoire. In: Circulations et identités culturelles alpines à la fin de la Préhistoire. Matériaux pour une étude. Programme CIRCALP 1997-1998. Travaux du Centre d'Archéologie Préhistorique de Valence, vol. 2, 1999, pp. 77-114.

Broglio 1994 : BROGLIO (A.) - Moutain sites in the context of North-East italian Upper Palaeolithic and Mesolithic. Preistoria Alpina, 28, 1994, pp. 93-148.

Court-Picon 2003 : COURT-PICON (M) - Approches palynologique et dendrochronologique de la mise en place du paysage dans le Champsaur (Hautes-Alpes, France) à l'interface des dynamiques naturelles et des dynamiques sociales. Thématique, méthodologie et premiers résultats. Archéologie du Midi Médiéval, 21, 2003, pp. 211-224.

Curdy 2002 : CURDY (Ph.) dir. - Premiers hommes dans les Alpes de 50000 à 5000 avant Jésus-Christ. Lausanne, Musées cantonaux du Valais, Sion, éd. Payot, 2002, 200 p.

Curdy et al. 1999 : CURDY (Ph.), DAVID-ELBIALI (M.), HONEGGER (M.) Le peuplement du Mésolithique à la fin de l'âge du Fer dans les Alpes Suisse occidentales. In: DELLA CASA (Ph.) ed. - Prehistoric Alpine Environment, society and economy. Bonn, Rudolf Habelt GmbG, 1999, pp. 47-59.

Di Pillo 1999 : DI PILLO (M.) - Settlement patterns in the Upper Adige basin from the Final Bronze âge. Preistoria Alpina, 35, 1999, pp. 93-103.

Édouard et al. 1991 : ÉDOUARD (J.-L.), TESSIER (L.), THOMAS (A.) Limite supérieure de la forêt au cours de 1'Holocène dans les Alpes françaises. Dendrochronologia, 9, 1991, pp. 125-142.

Fedele 1990 : FEDELE (F.) - Prehistoric and ancient man at higher altitudes ans latitudes. European mountains: the Alps. In: Impact of the prehistoric and medieval man on the vegetation: man at the forest limit. Strasbourg, 1990, pp. 25-29.

Frachetti, Chippindale 2002 : FRACHETTI (M.), CHIPPINDALE (C.) Alpine imagery, Alpine space, Alpine time; and prehistoric human experience. In : NASH (G.), CHIPPINDALE (C.) ed. - European Landscapes of Rock-Art. London, Routledge, 2002, pp. 116-143.

Galop 1998 : GALOP(D.) - La forêt, l'Homme et le troupeau dans les Pyrénées. 6000 ans d'histoire de l'environnement entre Garonne et Méditerranée. Toulouse, GEODE - Laboratoire d'Écologie terrestre - FRAMESPA, $1998,285 \mathrm{p}$.

Galop et al. 2003 : GALOP (D.), MAZIER (F.), LOPEZ-SAEZ (J.-A.), VANNIERE (B.) - Palynologie et histoire des activités humaines en milieu montagnard. Bilan provisoire des recherches et nouvelles orientations méthodologiques sur le versant nord des Pyrénées. Archéologie du Midi Médiéval, 21, 2003, pp. 159-170.

Garcia 1995 : GARCIA (D.) - Le peuplement protohistorique, les âges du Bronze et du Fer dans les Hautes-Alpes. In: GANET (I.) éd. - Les Hautes- 
Alpes. Carte Archéologique de la Gaule, Département des Hautes-Alpes. Paris, Maison des Sciences de l'Homme, 1995, pp. 40-41.

Ghesquière, Marcigny 1997 : GHESQUIÈRE (E.), MARCIGNY (C.) Le silex à l'âge du Bronze. L'industrie lithique à l'âge du Bronze du site de 1'île Tatihou à Saint-Vaast-la-Hougue (Manche). Revue Archéologique de l'Ouest, 14, 1997, pp. 27-47.

Haussmann 1998 : HAUSSMANN (L.) - L'âge du Bronze dans les HautesAlpes. Bulletin d'Études Préhistoriques et archéologiques alpines, Société Valdôtaine de Préhistoire et d'Archéologie, VII-VIII, Aoste, 1996-1997, 1998, pp. 97-135.

Jorda 1988 : JORDA (M.) - Modalités paléoclimatiques et chronologiques de la déglaciation würmienne dans les Alpes françaises du Sud (bassin durancien et Alpes-de-Haute-Provence). Bulletin de l'Association Française pour l'Étude du Quaternaire, 2/3, 1988, pp. 111-122.

Jorda 1991 : JORDA (M.) - Un milieu naturel montagnard et des hommes: lectures du paysage haut-alpin. In: Archéologie dans les Hautes-Alpes. Gap, Musée départemental, 1991, pp. 33- 50

Kaufmann, Nothdurfter 1997 : KAUFMANN (G.), NORTHDURFTER (H.) Annenburg, altitude settlements dating back to the Bronze âge in Val Venosta (BZ). Preistoria Alpina, 33, 1997, pp. 147-149.

Kharbourch, Gauthier 2000 : KHARBOUCH (M.), GAUTHIER (A.) Nouvelles analyses polliniques dans la région de la Vallée des Merveilles. Étude du Lac Long Inférieur (Tende, Alpes-Maritimes). Quaternaire, 11/3-4, 2000, pp. 243-256.

Küster 1994 : KUSTER (H.) - The economic use of Abies wood as timber in central Europe during Roman times. Vegetation History and Archaeobotany, 3, 1994, pp. 25-32.

Leveau 2003 : LEVEAU $(\mathrm{Ph}$.$) - Les recherches sur la montagne haut-alpine à$ la Maison Méditerranéenne des Sciences de l'Homme. Archéologie du Midi Médiéval, 21, 2003, pp. 183-184.

Leveau, Beaulieu 2001 : LEVEAU (Ph.), BEAULIEU (J.-L.) (de) - Forêt et pastoralisme dans les Alpes du Sud du Tardiglaciaire à l'époque actuelle à l'interface des dynamiques naturelles et des dynamiques sociales. In: BOËTSCH (G.) dir. - Évolutions biologiques et culturelles en milieu alpin. Actes de l'Université d'été 2000. CRDP de 1'Académie d'AixMarseille, 2001, pp. 37-53.

López-Sáez et al. 1998 : LÓPEZ-SÁEZ (J.A.), VAN GEEL (B.), FARBOSTEXIER (S.), DIOT (M.-F.) - Remarques paléoécologiques à propos de quelques palynomorphes non-polliniques provenant de sédiments quaternaires en France. Revue de Paléobiologie de Genève, 17, 1998, pp. 445-459.

Lumley 1995 : LUMLEY (H.) (de) - Le Grandiose et le Sacré. Aix-enProvence, Édisud, 1995.

Mocci et al. à paraître: MOCCI (Fl.), PALET-MARTINEZ (J.), SEGARD (M.), TZORTZIS (S.), WALSH (K.) - Peuplement, pastoralisme et modes d'exploitation de la moyenne et haute montagne depuis la Préhistoire dans le Parc national des Écrins (vallées du haut Champsaur et de Freissinières, Hautes-Alpes). In: BOUET (A.), VERDIN (F.) dir. - Mélanges offerts à Ph. Leveau. Université de Bordeaux III, éditions Ausonius, 20 p., à paraître.

Morin 2000 : MORIN (A.) - État documentaire sur le Néolithique final dans la zone préalpine: massifs et piémonts de la Chartreuse, du Vercors, du Buëch. In: Les Paléoalpins, Hommage à Pierre Bintz. Géologie alpine, 31, 2000, pp. 211-229.

Morin à paraître: MORIN (A.) avec la collaboration de CHAFFENET (G.), FAURE (H.), PELLETIER (D.), BRESSY (C.), SARGIANO (J.-P.) - Les ambiances culturelles néolithiques "haut alpines" et leurs insertions dans les chronologies du bassin rhodanien. In: Vie, culture et société dans les Alpes. Bilan des connaissances historiques des Alpes, des Hautes-Alpes et de Gap, Colloque International d'Histoire et de l'Archéologie de l'Arc alpin, Gap, septembre 2002, à paraître.

Morin et al. 2004 : MORIN (A.), BRESSY (C.), SIVAN (O.), BOUTTERIN (C.) - Vallée du Céans, Laragnais. Bilan Scientifique 2003, Service Régional de 1'Archéologie PACA, 2004, pp. 47-53.

Morin et al. 2005 : MORIN (A.), PICAVET (R.), BELLOT-GURLET (L.) Serrois- osanais et Bochaîne. Bilan Scientifique 2004, Service Régional de l'Archéologie PACA, 2005, pp. 63-64.
Muret 1991 : MURET (A.) - Huit campagnes de fouilles archéologiques au lieu-dit "Coumbauche" au col des Tourettes (Montmorin, Hautes-Alpes) In: Archéologie dans les Hautes-Alpes. Gap, Musée départemental, 1991, pp. 81-88

Nakagawa 1998 : NAKAGAWA (T.) - Études palynologiques dans les Alpes françaises centrales et méridionales: histoire de la végétation tardiglaciaire et holocène. Thèse de doctorat, Université de Droit, d'Économie et des Sciences d'Aix-Marseille III, Faculté des Sciences et Techniques de Saint-Jérôme, 1998, 211 p.

Nakagawa et al. 2000 : NAKAGAWA (T.), BEAULIEU (J.-L.) (de), KITAGAWA (H.) - Pollen-derived history of timber exploitation from the Roman period onwards in the Romanche valley, central French Alps. Vegetation History and Archaeobotany, 9, 2000, pp. 85-89.

Ozanne, Vital 1999 : OZANNE (J.-C.), VITAL (J.) - Documents pour servir à l'identification des relations transalpines occidentales dans la seconde moitié de l'âge du Bronze. Circulations et identités culturelles alpines à la fin de la Préhistoire - Matériaux pour une étude. Programme CIRCALP $1997-$ 1998. In: BEECHING (A.) - Circulations et identités culturelles alpines à la fin de la Préhistoire - Matériaux pour une étude, Programme CIRCALP 1997-1998. Travaux du Centre Archéologie Préhistorique de Valence, vol. 2, 1999, pp. 519-529.

Palet et al. 2003 : PALET (J.-M.), RICOU (F.), SEGARD (M.) - Prospections et sondages sur les sites d'altitude en Champsaur (Alpes du sud). Archéologie du Midi Médiéval, 21, 2003, pp. 199-210.

Palet-Martinez 2000 : PALET-MARTINEZ (J.M.) - Sondages archéologiques sur les structures pastorales des sites de Pré Brunet, Lac de Lauzons et Cabanes de Rougnous (Parc National des Écrins). Document Final de Synthèse, Service Régional de l'Archéologie PACA, Aix-en-Provence, 2000.

Palet-Martinez, Segard 2002 : PALET-MARTINEZ (J.M.), SEGARD (M.) Sondages archéologiques sur les structures pastorales des sites de Col du Palastre, Clot Lamiande II et Jas du Cros (Parc National des Écrins). Document Final de Synthèse, Service Régional de l'Archéologie PACA, Aix-en-Provence, 2002.

Palet-Martinez et al. 2003 : PALET-MARTINEZ (J.M.), RICOU (F.), SEGARD (M.) - Prospections et sondages sur les sites d'altitude en Champsaur (Alpes du Sud). Archéologie du Midi Médiéval, 21, 2003, pp. 199-210.

Pion 2004 : PION (G.) - Magdalénien, Épipaléolithique et Mésolithique ancien au Tardiglaciaire dans les deux Savoie et le Jura méridional. Thèse de $3^{\mathrm{e}}$ cycle sous la dir. d'Alain Daubigney, Université de Besançon, 2004, 2 vol., 479 p.

Ponel et al. 1992 : PONEL (Ph.), BEAULIEU (J.-L.) (de), TOBOLSKI (K.) Holocene paleoenvironments at the timberline in the Taillefer Massif, French Alps: a study of pollen, plant macrofossils and fossil insects. The Holocene, 2 (2), 1992, pp. 117-130.

Pons, Largarrigue 2003 : PONS (F.), LARGARRIGUE (A.) - Un habitat de plein air du Bronze ancien dans le Midi toulousain à Blagnac (Haute Garonne): le site de Cassagna 3. DocAMérid, 26, 2003, pp. 7-41.

Pothin 2000 : POTHIN (A.) - Impact de l'homme dans les milieux montagnards : Analyse pollinique de la séquence du Laux (alt.: 1108 m, Champsaur, Hautes-Alpes). DEA, Université de Droit, d'Économie et des Sciences d'Aix-Marseille III, Faculté des Sciences et Techniques de Saint-Jérôme, 2000,38 p.

Primas 1992 : PRIMAS (M.) - Intensification: le paradigme alpin. In: MORDANT (C.), RICHARD (A.) éd. - L'habitat et l'occupation du sol à l'âge du Bronze en Europe. Actes du colloque international de Lons-leSaunier, 16-19 mai 1990. Paris, CTHS, 1992, pp. 349-358 (Documents Préhistoriques, 4).

Py 2002 : PY (V.) - Modalités d'exploitation forestière dans la Haute Vallée de la Durance au Moyen Âge: approches croisées des données historiques, archéologiques et anthracologiques. Mémoire de DEA d'Archéologie Médiévale, Université de Provence, Aix-en-Provence, 2002.

Rendu et al. 1995 : RENDU (C.), CAMPMAJO (P.), DAVASSE (B.), GALOP(D.), CRABOL (D.) - Premières traces d'occupation pastorale sur la montagne d'Enveig. Travaux de Préhistoire Catalane, 8, 1992-95, pp. 35-43. 
Rousset et al. 1976 : ROUSSET (C.), DUBAR (M.), POIZAT (M.) - Les glaciers quaternaires dans les Alpes du Sud. In: la Préhistoire française, t. I, pp. 27-31.

Segard et al. 2003 : SEGARD (M.), WALSH (K.), COURT-PICON (M.), avec la collaboration de MOCCI (F.), PALET-MARTINEZ (J.) - L'occupation de la haute montagne dans les Alpes occidentales. Apport de l'archéologie et des analyses paléoenvironnementales. In: BOËTSCH (G.), DEVRIENDT (W.), PIGUEL (A.) dir. - Permanence et changements dans les sociétés alpines. Actes du colloque de Gap, juillet 2002. Aix-en-Provence, Édisud, 2003, pp. 17-30.

Talon 1997 : TALON (B.) - Évolution des zones supra-forestière des Alpes sudoccidentales françaises au cours de l'holocène. Analyse pédoanthracologique. Thèse de doctorat, Université d'Aix-Marseille III, 1997, 213 p.

Talon 2000 : TALON (B.) - Analyse anthracologique du site de Faravel VIIId. In: WALSH (K.), MOCCI (Fl.) - Fouille archéologique programmée sur la structure pastorale de Faravel XII, commune de Freissinières (05). Document Final de Synthèse, SRA PACA - Centre Camille Jullian - Parc national des Écrins, Aix-en-Provence, 2000, 44 p.

Talon 2003 : TALON (B.) - Analyse des charbons de bois des sites archéologiques de Chichin II et Chichin III. In : WALSH (K.), MOCCI (Fl.) - Prospectioninventaire diachronique et sondages archéologiques sur les sites d'altitude de Chichin II et III dans la haute vallée de Chichin (Freissinières, 05). Document Final de Synthèse, SRA PACA - Centre Camille Jullian - Parc national des Écrins, Aix-en-Provence, 2003, 90 p.

Talon et al. 1998 : TALON (B.), CARCAILLET (C.), THINON (M.) Pedoanthracological Studies of the Upper Tree-Limit Change During the Holocene in the French Alps. Géographie Physique et Quaternaire, 52 (2), 1998, pp. 195-208.

Tessier et al. 1993 : TESSIER (L.), BEAULIEU (J.-L.) (de), COUTEAUX (M.), ÉDOUARD (J.-L.), PONEL (Ph.), ROLANDO (C.), THINON (M.), THOMAS (A.), TOBOLSKI (K.) - Holocene palaeoenvironments at the timberline in the French Alps - a multisciplinary approach. Boreas, 22, 1993, pp. 244-254.

Thinon, Talon 1998 : THINON (B.), TALON (M.) - Ampleur de l'anthropisation des étages supérieurs dans les Alpes du sud: données pédoanthracologiques. Écologie, 29 (1-2), 1998, pp. 323-328.

Tzortzis 2001 : TZORTZIS (S.) - Premières données sur l'industrie lithique du site de Faravel XIII, In: WALSH (K.), MOCCI (Fl.) - Fouille archéologique programmée sur sites d'altitude de Faravel XIII et Faravel XIV, commune de Freissinières (05). Document Final de Synthèse, SRA PACA - Centre Camille Jullian - Parc national des Écrins, Aix-en-Provence, 2001, 72 p.

Tzortzis 2002 : TZORTZIS (S.) - Notes préliminaires sur les séries lithiques des sites de Faravel XVIII, XI et XXII et des sites de la haute vallée de Chichin. In: WALSH (K.), MOCCI (Fl.) - Fouille archéologique programmée sur sites d'altitude de Faravel XIII et Faravel XIV, commune de Freissinières (05). Document Final de Synthèse, SRA PACA - Centre Camille Jullian Parc national des Écrins, Aix-en-Provence, 2002, 72 p.

Tzortzis 2003 : TZORTZIS (S.) - Note préliminaire sur les séries lithiques des sites de la haute vallée de Chichin (Chichin II, III, IV et V) et dans la vallée du Fournel. In: WALSH (K.), MOCCI (Fl.) - Prospection-inventaire diachronique et sondages archéologiques sur les sites d'altitude de Chichin II et III dans la haute vallée de Chichin (Freissinières, 05). Document Final de Synthèse, SRA PACA - Centre Camille Jullian - Parc national des Écrins, Aix-en-Provence, 2003, 90 p.

Vannière 2001 : VANNIERE (B.) - Feu, agro-pastoralisme et dynamiques environnementales en France durant l'Holocène. Analyse du signal incendie, approches sédimentologiques et études de cas en Berry, Pyrénées et Franche-Comté. Thèse de 1'Institut National Agronomique de ParisGrignon, 2001, 329 p.

Vannière $\boldsymbol{e t}$ al. 2001 : VANNIÈRE (B.), GALOP (D.), RENDU (C.), DAVASSE (B.) - Feu et pratiques agro-pastorales dans les Pyrénées-Orientales: le cas de la montagne d'Enveig (Cerdagne, P.-O., France). Sud-Ouest Européen, 11, 2001, pp. 29-42.

Walsh 1999 : WALSH $(\mathrm{K})$ - Sondages archéologiques sur les structures pastorales de Faravel VIIId et XIII, juillet 1999, commune de Freissinières (05). Document Final de Synthèse, SRA PACA - Centre Camille Jullian - Parc national des Écrins, Aix-en-Provence, 1999, 31 p.

Walsh 2001 : WALSH (K.) - Fouille archéologique programmée sur sites d'altitude de Faravel XIII et Faravel XIV, commune de Freissinières (05). Document Final de Synthèse, SRA PACA - Centre Camille Jullian - Parc national des Écrins, Aix-en-Provence, 2001, 72 p.

Walsh 2002 : WALSH (K.) - Sondages archéologiques sur trois sites d'altitude de la Montagne de Faravel et de l'Abri Fangeas: Faravel XIX, Fangeas VI et Fangeas VII et prospection-inventaire dans la haute vallée de Chichin (05). Document Final de Synthèse, SRA PACA - Centre Camille Jullian - Parc national des Écrins, Aix-en-Provence, 2001, $137 \mathrm{p}$.

Walsh 2005 : WALSH (K.) - Risk and marginality at high altitudes: new interpretations from fieldwork on the Faravel Plateau, Hautes-Alpes. Antiquity, 79, 304, 2005, pp. 289-305.

Walsh, Mocci 2003a: WALSH (K.), MOCCI (Fl.), avec la collaboration de DUMAS (V.), DURAND (A.), TALON (B.), TZORTZIS (S.) - 9000 ans d'occupation du sol en moyenne et haute montagne: la vallée de Freissinières dans le Parc national des Écrins (Freissinières, Hautes-Alpes). Archéologie du Midi Médiéval, 21, 2003, pp. 185-198.

Walsh, Mocci 2003b: WALSH (K.), MOCCI (Fl.) - Prospection-inventaire 